\title{
VIOLENCE AGAINST EDUCATORS
}

\author{
A Thesis \\ Submitted to the Faculty of the Graduate School \\ University of Minnesota \\ By
}

Chia Wei

In Partial Fulfillment of the Requirements for the Degree of Master of Science

Susan Goodwin Gerberich

September 2011 
(C) Chia Wei 2011 


\section{ACKNOWLEDGMENTS}

My kindest regards to Dr. Susan Gerberich, for her continued academic research advising and role as an inspirational mentor. Also, to Andy Ryan, for his statistical expertise contributions, the Nancy A. Robertson Endowed Graduate Fellowship in Injury Prevention, and Drs. Alexander, Nachreiner and Chaplin, who also participated in my graduate committee.

I would also like to thank my family and friends for all their support while there has been 7,137 miles between us. Thank you my dearest Dad, Mom, Chi and Chiao for giving me faith and strength through all my struggles and accomplishments while pursing my Master's degree in a foreign country. Your encouragement has and will always make me feel like I can achieve all of my dreams. A special thanks to Sohail, whose dedication is nothing short of invaluable. 


\section{Abstract}

\section{Introduction}

Physical and non-physical violence are recognized as a major public health issue in the work environment and one commonly ignored population that may experience work-related violence is teachers/educators. However, to date, there has been no apparent investigation to examine the relations between various exposures of interest and rates of violence, based on hours worked by educators. The objective of the current study was to identify the potential risks for both physical assault (PA) and nonphysical violence (NPV) against licensed Minnesota educators, based on hours exposed.

\section{Methods}

The target study population was licensed kindergarten through grade 12 educators in Minnesota, who were identified in the Minnesota Department of Education database as of 2003. A random sample of 26,000 educators was selected from this database to determine eligibility; 6,469 eligible participants were included in the study. Data were collected between April 27, 2005 and March 31, 2006, using specially designed mailed questionnaires. PA and NPV event data were collected from respondents for a 12-month time period prior to their respective survey completion dates. Injury rates per 100,000 working hours were calculated, using generalized linear models with Poisson distribution for both PA and NPV events among educators. Multivariate analyses utilized calculated rate ratios to determine the strength of the association between exposures of interest and injury outcomes (PA and NPV); selection of confounders for multiple regression with Poisson distribution was based on Directed Acyclic Graphs, following the methods described 
by Greenland and colleagues.

\section{Results}

Among all eligible respondents, the majority was female (77\%). The total NPV rates were higher than the total PA rates (26.37 and 5.31, respectively). For the four subcategories of NPV, the total rates were: threat (34.82); sexual harassment (7.58); verbal abuse (55.48); and bullying (19.62).

Multivariate analyses for respective PA and NPV models revealed increased rate ratios for: educators who were not married $(1.28,1.20)$ versus married; worked in public alternative $(1.73,1.93)$, compared to public schools; those who worked in special education $(4.39,1.45)$ and in multiple activities $(4.01,1.41)$, compared with standard classroom teaching; and worked with class sizes less than ten $(2.71,1.43)$, compared to ten to $<25$ students. Decreased risks for respective PA and NPV models were identified for: males $(0.73,0.85)$, compared with females; those who worked as educators for $20-29(0.66,0.66)$, and $>30(0.55,0.60)$ years, compared to less than ten years; and worked in their current school for more than $20(0.39,0.80)$ versus less than five years.

\section{Conclusions}

Results from this study provided information about factors associated with increased and decreased risks for violence against educators, based on hours exposed. This information provides a basis for further investigation and consideration of possible intervention strategies to reduce violence in the school environment.

Key Words: Occupational violence; work-related violence; physical assault; nonphysical violence, risk factors 


\section{Table of Contents}

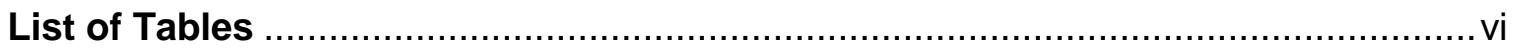

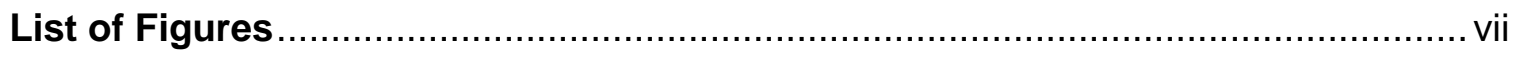

Plan a Master's Thesis Contract ........................................................... viii

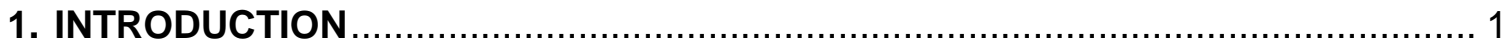

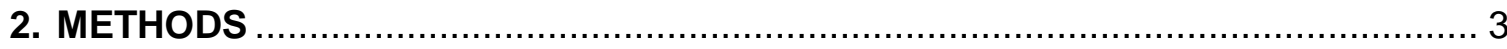

2.1 Study Population and Study Cohort ....................................................... 3

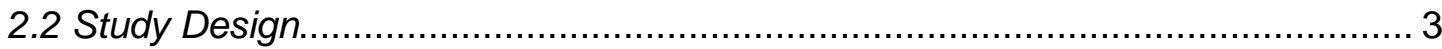

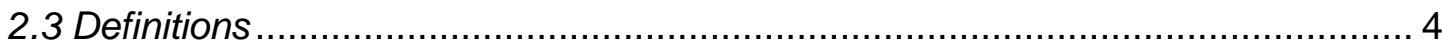

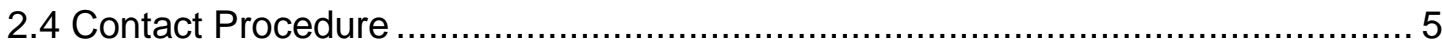

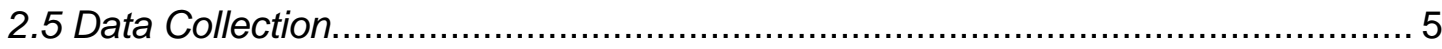

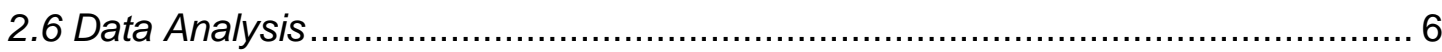

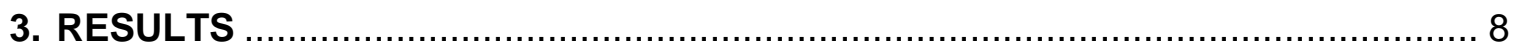

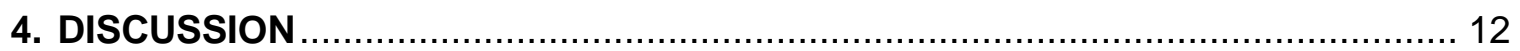

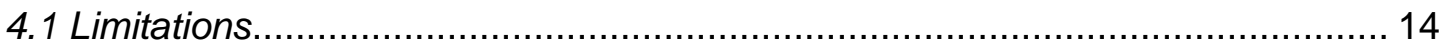

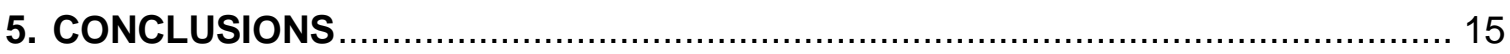

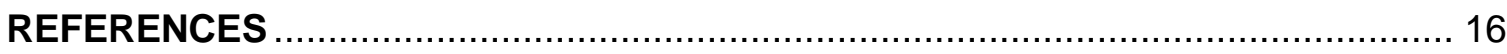

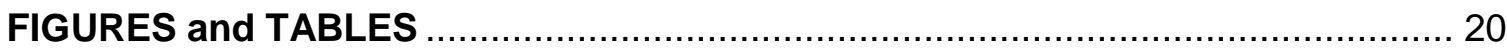

Figure1. Minnesota Educators' Study- Phase 1: Response Numbers................... 21

Figure2. Directed Acyclic Graph Example .............................................. 22

Table 1a. Physical Assault and Non-Physical Violence Rates Per 100,000

Working Hours Per Year: Violence Against Educators Study ............................ 23

Table 1a. (continued) Physical Assault and Non-Physical Violence Rates Per 100,000 Working Hours Per Year: Violence Against Educators Study 
Table 1b. Non-Physical Violence Rates Per 100,000 Working Hours Per Year:

Violence Against Educators Study

Table 1b. (continued) Non-Physical Violence Rates Per 100,000 Working Hours Per Year: Violence Against Educators Study

Table 2a. Rate Ratios for Physical Assault and Non-Physical Violence Rates Per 100,000 Working Hours Per Year: Violence Against Educators Study

Table 2a. (continued) Rate Ratios for Physical Assault and Non-Physical

Violence Rates Per 100,000 Working Hours Per Year: Violence Against

Educators Study

Table 2b. Rate Ratios for Non-Physical Violence Rates Per 100,000 Working Hours Per Year: Violence Against Educators Study 29

Table 2b. (continued) Rate Ratios for Non-Physical Violence Rates Per 100,000 Working Hours Per Year: Violence Against Educators Study 30

Appendices 31

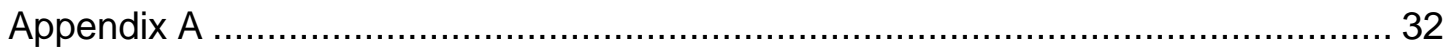

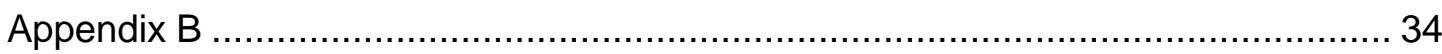




\section{List of Tables}

Table 1a. Physical Assault and Non-Physical Violence Rates Per 100,000

Working Hours Per Year: Violence Against Educators Study

Table 1a. (continued) Physical Assault and Non-Physical Violence Rates Per 100,000 Working Hours Per Year: Violence Against Educators Study

Table 1b. Non-Physical Violence Rates Per 100,000 Working Hours Per Year:

Violence Against Educators Study

Table 1b. (continued) Non-Physical Violence Rates Per 100,000 Working Hours

Per Year: Violence Against Educators Study

Table 2a. Rate Ratios for Physical Assault and Non-Physical Violence Rates Per 100,000 Working Hours Per Year: Violence Against Educators Study

Table 2a. (continued) Rate Ratios for Physical Assault and Non-Physical

Violence Rates Per 100,000 Working Hours Per Year: Violence Against

Educators Study

Table 2b. Rate Ratios for Non-Physical Violence Rates Per 100,000 Working Hours Per Year: Violence Against Educators Study

Table 2b. (continued) Rate Ratios for Non-Physical Violence Rates Per 100,000

Working Hours Per Year: Violence Against Educators Study 30 


\section{List of Figures}

Figure1. Minnesota Educators' Study- Phase 1: Response Numbers................... 21

Figure2. Directed Acyclic Graph Example ............................................. 22 


\section{Plan a Master's Thesis Contract}

The contract for this project involved completion of a manuscript prepared for publication in a peer-reviewed journal. This project involved descriptive and multivariate analyses of data pertinent to work-related violence among educators in Minnesota.

To fulfill this contract, the following were completed:

1. Comprehensive literature review;

2. Analysis and interpretation of the data;

3. Preparation of the manuscript.

M.S. committee members included:

Susan G. Gerberich, Ph.D.

Bruce H. Alexander, Ph.D.

Nancy M. Nachreiner, Ph.D.

Jonathan Chaplin, Ph.D. 


\section{INTRODUCTION}

Physical and non-physical violence had been recognized as a major public health issue in the work environment (Rosenberg and Fenley, 1991). According to the 2001 National Crime Victimization Survey (NCVS), there was an average of 1.7 million people who experienced violent crimes while working or on duty in the United States (U.S.) (Duhart, 2001). One commonly ignored population that has a high risk of workrelated violence is teachers/educators (Duhart, 2001; DeVoe et al, 2005; Dinkes et al., 2007). In particular, educators reportedly have a higher risk for simple assault (an attack without a weapon) than aggravated assault (attack with a weapon) (Duhart, 2001).

Between 1999 and 2003, it was estimated that 183,000 teachers were victims of non-fatal crimes at schools, accounting for an annual rate of 39 crimes per 1,000 teachers (DeVoe et al., 2005). Furthermore, students play an important role in violence against educators as they have been identified as the primary perpetrators (Peek-Asa et al., 1998; Hashemi and Webster, 1998; Fisher and Kettl, 2003; DeVoe et al., 2005; Levin et al., 2006). It has also been reported that risk of school workrelated violence varies across geographical locations; teachers working in city versus suburban or rural schools were more likely to have been physically attacked or threatened with injury by students (Dinkes et al., 2007). In addition to location, the grade level may also be associated with violence rates: U.S. junior high school teachers had a higher annual work-related violence rate per 1,000 teachers (54.2) than high school (38.1) and elementary school teachers (16.8); however, special education teachers had the highest rate among all teaching categories (68.4) (Duhart, 2001). It has been reported that violence affects teachers' performance in 
class and the quality of teaching; retention of educators is a major concern in such high-risk occupational environments (Fisher and Kettl, 2003).

To date, there has been no apparent investigation to examine the relations between various exposures of interest and rates of occupational violence, based on hours worked by educators. The objective of the current study was to identify the potential risk for both physical assault (PA) and nonphysical violence (NPV) against licensed Minnesota educators, based on hours exposed. 


\section{METHODS}

\subsection{Study Population and Study Cohort}

The target study population was licensed Kindergarten through Grade 12 (K-12) educators in Minnesota, who were identified in the Minnesota Department of Education database as of 2003. This study population was selected because of the ability to contact through home versus workplace addresses, and the availability of various demographic information including: year of birth; fiscal year data; zip code; principal and teacher years; salary; teaching time; grade level(s) taught; license type; and educational level.

There were originally 320,333 licensed educators in the database. After eliminating educators who were deceased, not licensed, or no longer working in the year prior to 2003 , a random sample of 26,000 educators was selected for screening to determine eligibility.

\subsection{Study Design}

The study cohort included educators who worked as licensed professionals (K-12) in Minnesota during a 12-month period prior to survey completion. The study entailed two phases; this paper focused only on Phase 1, which was designed to determine the frequency of work-related violence, and to identify the potential risk for both PA and NPV against educators in Minnesota, based on hours exposed.

Using a random number generator, 300 educators were randomly selected from 320,333 licensed educators in the Minnesota Department of Education Database to participate in an initial pilot study to test the methods and data collection instruments. From the pilot study, it was discovered that there was a large proportion of educators listed in the database who were deceased, not licensed, or no longer working in the 
year prior to 2003; these were eliminated. Thus, in order to identify the eligibility of the remaining license list before implementation of the comprehensive study, a random sample of 26,000 educators received an eligibility screener; 8,614 (33\%) educators responded to the screener, resulting in 6,469 (75\%) apparently eligible responders who indicated working in Minnesota during the previous 12 months. Subsequently, data were collected between April 27, 2005 and March 31, 2006. Figure 1 shows the response diagram.

\subsection{Definitions}

Work-related violence includes physical assault (PA) and nonphysical violence (NPV), involving acts that use intentional physical force or emotional abuse with the potential for causing physical or emotional injury and consequences against an employee. In this study, work-related violence included any events or activities that were associated with and occurred in the educator's work-environment, including work-related travel.

PA occurred when educators were hit, slapped, kicked, pushed, choked, grabbed, sexually assaulted, or otherwise subjected to physical contact intended to injure or harm. NPV included threats, sexual harassment, verbal abuse, and bullying. Threat was defined as when someone used words, gestures, or actions with the intent of intimidating, frightening, or harming them (physically or otherwise). Sexual harassment occurred when educators experienced any type of unwelcome sexual behavior (words or actions) that created a hostile work environment. Verbal abuse occurred when another person yelled or swore at the educator, called the educator names, or used other words intended to control or hurt. Bullying was defined as repeated acts of intimidation or coercion. 


\subsection{Contact Procedure}

Approval to conduct this study was obtained from the Institutional Review Board at the University of Minnesota. An initial survey packet, sent to the 6,469 eligible educators, included: a specially designed survey instrument; a letter inviting participation; informed consent information; and a postage-paid, return envelope. Participants were involved in this study by returning a completed questionnaire. For those who did not respond, up to four mailings were sent to increase the response rate. A final packet was sent to remaining non-respondents; this included a one-page survey to collect important demographic and work-related violent event information.

\subsection{Data Collection}

The comprehensive survey data were collected via specially designed mailed questionnaires. Of those 6,469 educators who were mailed a survey packet, 5,038 (78\%) returned a fully completed survey and 377 returned a short survey. As a result, a total of 4,731 eligible educators were included in the study analysis.

The comprehensive instrument enabled collection of data pertinent to the occurrence of PA and NPV events, determination of the months in which they worked, and relevant exposures in the previous 12-month period. The number of events was established per educator and although educators may have experienced more than one violent event (physical or nonphysical), only one event was included in the current analysis.

Further data collected included the educator's demographic information: gender; age; race; ethnic classification; marital status; educational information; years of experience and job classification (full/part-time, substitution, etc.); characteristics of the teaching environment -- such as type of school (public, private, etc.); educators' 
activity (classroom teacher, administrator, etc.); class size; and grade levels. Finally, working hours and number of days worked per week were also collected.

\subsection{Data Analysis}

Data analysis commenced with descriptive statistics including, number of reported events, and calculation of injury rates and rate ratios per 100,000 working hours.

Injury rates per 100,000 working hours were calculated, using generalized linear models with Poisson distribution for both PA and NPV events among educators; missing working hours were addressed through imputation (Madow et al., 1983). This imputation was accomplished by using average hours worked per month by educators from the same job classification (full-time/part-time contract or substitute categories). Multivariable regression models with Poisson distribution incorporated calculated rate ratios to determine the strength of the association between exposures of interest and injury outcomes (PA and NPV).

Potential selection bias, resulting from different response patterns, was examined by inversely weighting observed responses by probabilities of response, estimated as a function of characteristics available from the licensing database: characteristics included: gender; zip code; year of birth; first and last year of licensure; last fiscal year of employment; years worked as a teacher/principal; salary; class period minutes; class periods per week and grade levels taught. This method re-weights estimates using group response characteristics to account for potential differences in responses (Horvitz and Thompson, 1952). The probability of eligibility of nonrespondents was estimated from these same factors. (Mongin, 2001)

A causal model was developed to determine the variables to be measured and 
controlled for the overall study analyses. From this model, individual Directed Acyclic Graphs (DAGs) were derived to select the minimum set of potential confounding factors for each exposure of interest (Greenland et al., 1999; Hernan et al., 2002). An example of a DAG for one exposure of interest (grade taught) is shown in Figure 2. 


\section{RESULTS}

A total of 4,731 eligible educators, who worked as licensed K-12 educators in Minnesota during the prior 12 months, were included in this analysis. According to gender, these respondents included 3,628 (76.7\%) female and 1,103 (23.3\%) male educators.

Tables $1 \mathrm{a}$ and $1 \mathrm{~b}$ present the numbers of responses, numbers of events, and injury rates per 100,000 working hours per year for physical assault (PA) and nonphysical violence (NPV). As identified, the total NPV rate is higher than the total PA rate (26.37 and 5.31, respectively). Women had slightly higher rates than men for both PA and NPV. By age, those $40-49$ years had the highest PA rate; for NPV, the rate was highest among those $60-79$ years. Hispanics, compared to non-Hispanics, had higher PA and NPV rates; by race, little difference was observed. According to marital status, those who were not married, versus married, had slightly higher PA and NPV rates. Among education classifications, those with an education specialist degree had the highest PA rate, while those with a master's degree had the highest NPV rate. Educators who had taught less than 10, versus 10 years or more, had slightly higher PA and NPV rates. Similarly, PA and NPV rates were higher among educators who worked in the current school for less than five years. These rates were also higher for educators who taught in public alternative schools, magnet, or multiple types of schools. By job classification, PA rates were higher for those working full time versus part-time and in various substitute categories while NPV rates were higher for part-time and substitute educators. Based on educators' activities, school social workers, psychologists, and special education teachers had the highest PA and NPV rates. It was also shown that class size may affect injury 
rates, especially classes with fewer than 10 students; however, larger class sizes were also important for NPV. Both PA and NPV rates were the highest for educators who taught in all grades; otherwise, PA rates were higher among those who taught kindergarten through grade 2 while NPV rates were higher in grades 7-9 and 10-12.

Table $1 \mathrm{~b}$ identifies total rates for the four subcategories of NPV. The total rates are as follows: threat (34.82); sexual harassment (7.58); verbal abuse (55.48); and bullying (19.62). Some variations are noted among these categories.

Results of multivariate analysis for respective PA and NPV models are shown in tables $2 \mathrm{a}$ and $2 \mathrm{~b}$. These results are presented as rate ratios per 100,000 working hours per year, based on the exposures of interest. As shown in table 2a, increased risks for both PA and NPV, respectively, were shown for: educators who were not married versus married $(1.28,1.20)$; worked in public alternative $(1.73,1.93)$, compared to public schools; those who worked in special education $(4.39,1.45)$ or in multiple activities $(4.01,1.41)$, compared with standard classroom teaching; and class sizes were less than ten students $(2.71,1.43)$, compared to ten to $<25$ students. Decreased risks for both PA and NPV were identified for: males compared with females $(0.73,0.85)$; those who worked as teachers for $20-29(0.66,0.66)$, and more than 30 years $(0.55,0.60)$ compared to less than ten years; worked in their current school for more than $20(0.39,0.80)$ versus less than five years; and worked in private parochial $(0.17,0.51)$ versus public schools.

Other increased risks for PA were identified for those educators who: held a master's degree (1.23), or an education specialist degree (1.53), versus an associate/bachelor's degree; taught in various school types (3.05), versus public schools; worked primarily as a social worker (4.50), versus a standard classroom 
teacher. Decreased risks were shown for PA when the class size was larger than 25 (0.54), versus ten to <25 students; and educators who frequently taught in grades 10 to 12 (0.39), versus kindergarten through grade 2. For NPV, risks were increased for educators: older than 60 (1.26), versus 50-59 years; who worked part-time (1.44) or in various substitute categories (1.73) versus full-time; whose class size was larger than 25 (1.16), versus 10-25 students; and those who taught in grades 3-6 (1.30), 79 (1.79), 10-12(1.76) and multiple grades (1.77), versus kindergarten to second grade. A decreased risk for NPV (0.83) was identified for those who worked as teachers for 10-19 years, compared to less than ten years.

Table $2 \mathrm{~b}$ shows rate ratios per 100,000 working hours per year for the NPV subcategories. For threat, increased risks were identified for educators: aged 30-39 (1.21) and 60-79 (1.34) years compared to 50-59; who worked in public alternative (1.36), compared to public schools; who worked in various substitute categories (2.18), versus full-time; and worked in special education (1.42) compared to standard classroom teaching. A decreased risk for threat was determined when class sizes were larger than $25(0.8)$, versus $10-25$ students. For sexual harassment, increased risks were shown for: age 20-29 (1.77), 30-39 (1.52), 40-49 (1.61), compared to 5059 years; those were not married (1.59) versus married; those with education specialist degrees (1.55) compared to associate/bachelor degrees; educators who taught in public alternative (1.93) and multiple school types (2.84), compared to public schools; and had classrooms with less than ten (1.6) compared to 10-25 students. Decreased risks were identified for sexual harassment among male (0.69), compared to female educators, and among those who worked for 10-19 years (0.58), versus less than ten years. In terms of verbal abuse, increased risks were seen in 
educators who worked: in public alternative (1.2), compared to public schools; and in part-time position (1.51) or various substitute categories (1.91) versus full-time. It was also noted that educators with a masters' $(0.87)$ or education specialist $(0.77)$, versus associated/bachelors' degrees, had decreased risks for verbal abuse. For bullying, increased risks were identified for educators who worked in part-time positions (1.54) or various substitute categories (2.03) versus full-time; a decreased risk was seen for male educators $(0.71)$ compared to female. 


\section{DISCUSSION}

This current study was based on a comprehensive population investigation of violence against licensed educators in Minnesota. As identified in this study, the overall rate per 100,000 working hours per year was much greater for NPV (26.37) than for PA (5.31) and the highest rate among all NPV subcategories was for verbal abuse. The majority of respondents were female who were more likely to experience PA and NPV compared to male educators. From other studies, using a variety of methods and populations, it has been reported that males were more likely than females educators to experience violence (Peek-Asa et al., 1998; DeVoe et al., 2005; Liss and McCaskell, 1994; Peek-Asa et al., 1997; Duhart, 2001).

A study conducted in Los Angeles reported that educators with a master's and/or doctoral degree were more likely to report an assault (Casteel et al., 2007). However, the current study only found those with a master's degree, versus associate/bachelor's degree, had an increased PA rate; further, those with an education specialist degree, compared with an associate/bachelor's degree, had increased risks for PA and sexual harassment (NPV). According to working experience, educators in the current study, with more years of teaching experience, had decreased PA and NPV rates; however, there was an opposite finding from another study, using a different study population, which suggested that teachers with more experience were more likely to report an assault (Casteel et al., 2007).

In terms of the work environment, educators who taught in public alternative, versus public schools, had increased risks for both PA and NPV, and decreased 
risks for those who taught in private compared to public schools -- findings consistent with previous reports (Dinkes et al., 2007; Nolle et al., 2007). Based on multivariate analysis, those educators who taught in classrooms with more than 25, versus 10 to less than 25 students, had decreased rates for PA but increased rates for NPV. Similarity, risks for educators who taught in grades 10-12, versus kindergarten through second grades, had decreased risks for PA and increased risks for NPV. These findings were consistent with a previous study, showing that secondary school educators were less likely to experience PA than elementary school teachers, but more likely to experience NPV (threat) (DeVoe et al., 2005).

It has been reported that violence directly impacts on teachers' performance in class and the quality of teaching (Fisher and Kettl, 2003). Work-related violence has been associated with consequences of work performance, including reduced productivity, reduced quality of life, increased turnover, and absenteeism (Lanza, 1983; Martino et al., 2003; Gerberich et al., 2004) as well as fear of recurrent assault, family disruption, and career changes (Liss and McCaaskell, 1994). Work-related violence has also been associated with short-term and long-term physical, emotional and professional effects (Levin et al., 1998; Gerberich et al., 2004). In particular, the impact of non-physical violence cannot be neglected since the consequences of nonphysical violence have been shown to be more severe than physical assault (Gerberich et al., 2004) and may lead to more advanced mental health problems such as acute stress disorder or post-traumatic stress syndrome (Brewin et al., 1999). Moreover, the resulting impact on work status (including restricted or modified work, quitting, transferring, and/or obtaining a leave of absence) for non-physical violence, versus physical assault, has also been shown to be greater (Gerberich et al., 2004). 


\subsection{Limitations}

Limitations in this study include the fact that data, pertinent to violence events and relevant exposures, were collected through self-report, which may involve potential biases. Numerous strategies to minimize these potential biases were incorporated; for example, recall bias, was minimized, to some degree, by limiting the recall of violent events to the previous 12 months. This approach has also been implemented in previous studies (Gabel and Gerberich, 2002; Gerberich et al., 2004, 2005; Sage et al., 2010). In addition, a calendar with relevant holidays was included in the questionnaire to assist participants' recall of events. Information bias was further addressed by follow-up via mail to clarify ambiguous and missing information. As previously noted, missing working hours were addressed through imputation (Madow et al,. 1983). No important differences were identified between imputed and non-imputed rates for PA and NPV: imputed working hours were used in the data analysis. Potential response bias was reduced by inversely weighting observed responses by probabilities of response, estimated as a function of educator characteristics available from the licensing database (Horvitz and Thompson, 1952). To account for unknown eligibility among non-respondents, probability of eligibility was estimated from these same factors (Mongin, 2001). Selection of confounders for multiple regression models was based on DAGs to reduce the effect of confounding (Greenland et al., 1999). Of note, is that the calculated rates may be underestimated, given that a maximum of one event was included per educator in the data analysis; yet, many educators experienced multiple violent events. 


\section{CONCLUSIONS}

This study enabled estimation of PA and NPV rates among educators who taught kindergarten through grades 12 , and provided information to determine the association between exposures of interest and these rates. In particular, findings in this study provided information about factors associated with increased and decreased risks for violence against educators that provide opportunities for further investigation and consideration of interventions to reduce violence in the school environment. Most importantly, this current study serves as the basis for future analytical studies and for development of appropriate prevention and control efforts in work-related violence.

\section{ACKNOWLEDGMENTS}

Support for this effort was provided, in part, by: the National Institute for Occupational Safety and Health (R01 OH 007816), Centers for Disease Control and Prevention, Department of Health and Human Services; the Center for Violence Prevention and Control and the Regional Injury Prevention Research Center, Division of Environmental Health Sciences, School of Public Health, University of Minnesota; and the Nancy A. Robertson Endowed Graduate Fellowship in Injury Prevention. The contents of this effort are solely the responsibility of the authors and do not necessarily represent the official view of the Centers for Disease Control and Prevention or other associated entities. We also wish to acknowledge the support of our Educational Advisory Consulting Team members who were integral to this study: Willarene Beasley, Charles Goodwin, Donald Hilts, Laura R. Langhoff, and Joseph P. Miller. 


\section{REFERENCES}

Brewin C.R., Andrews B., Rose S., Kirk M., 1999. Acute Stress Disorder and Posttraumatic Stress Disorder in Victims of Violent Crime. The American Journal of Psychiatry 156:360-366.

Casteel C., Peek-Asa C., Limbos M.A., 2007. Predictors of non-fatal assault injury to public school teachers in Los Angeles City. American Journal of Industrial Medicine 50(12):932-939.

DeVoe, J.F., Peter, K., Noonan, M., Snyder, T.D., Baum, K., 2005. Indicators of School Crime and Safety: 2005 (NCES 2006-001/NCJ 210697). U.S. Departments of Education and Justice, Washington, DC: U.S. Government Printing Office

Dinkes, R., Cataldi, E.F., and Lin-Kelly, W., 2007. Indicators of School Crime and Safety: 2007 (NCES 2008-021/NCJ 219553). National Center for Education Statistics, Institute of Education Sciences, U.S. Department of Education, and Bureau of Justice Statistics, Office of Justice Programs, U.S. Department of Justice. Washington, DC.

Duhart, D.T., 2001. Violence in the workplace, 1993-99, National Crime Victimization Survey, Bureau of Justice Statistics Special Report, U.S. Department of Justice, Office of Justice Programs.

Fisher K., Kettl P., 2003. Teachers' perceptions of school violence. Journal of Pediatric Health Care 17(2):79-83.

Gabel C.L., Gerberich S.G., 2002. Risk factors for injury among veterinarians. Epidemiology 13 (1):80-86. 
Greenland S., Pearl J., Robins J.M., 1999. Causal diagrams for epidemiology research. Epidemiology 10(1):37-48

Gerberich S.G., Church T.R., McGovern P.M., Hansen H.E., Nachreiner N.M., Geisser M.S., Ryan A.D., Mongin S.J., Watt G.D., 2004. An epidemiological study of the magnitude and consequences of work related violence: the Minnesota Nurses' Study. Occupational and Environmental Medicine 61(6):495-503

Gerberich S.G., Church T.R., McGovern P.M., Hansen H.E., Nachreiner N.M., Geisser M.S., Ryan A.D., Mongin S.J., Watt G.D., Jurek A., 2005. Risk factors for work-related assaults on nurses. Epidemiology 16(5):704-709.

Hashemi L., Webster B.S., 1998. Non-fatal workplace violence workers' compensation claims (1993-1996). Journal of Occupational and Environmental Medicine 40(6):561-567

Hernan M.A., Hernandez-Diaz S., Werler M.M., Mitchell A.A., 2002. Causal knowledge as a prerequisite for confounding evaluation: an application to birth defects epidemiology. American Journal Epidemiology 155(2):176-184.

Horvitz, D., Thompson, D., 1952. A generalization of sampling without replacement from a finite universe. American Statistical Association Journal 47, 663685.

Lanza, M.L., 1983. The Reactions of Nursing Staff to Physical Assault by a Patient. Hospital Community Psychiatry 34:44-47.

Levin P.F., Hewitt J.B., Misner S.T., 1999. Insights of Nurses about Assault in Hospital-based Emergency Departments. Image -- The Journal of Nursing 
Scholarship 30(3):249-254

Levin P.F., Martinez M.Q., Walcott-McQuigg J., Chen S.P., Amman M., Guenette C., 2006. Injuries associated with teacher assaults: Magnitude, nature, cost, and outcome. American Association of Occupational Health Nurses 54(5):210-216.

Liss GM, McCaskell L., 1994. Violence in the workplace. Canadian Medical Association Journal 151(9):1243-1246

Madow W.G., Nisselson H., Olkin I., 1983. Incomplete data in sample survey, Volume 1, Report and Case Studies. New York: Academic Press.

Martino V.D., Hoel H., Cooper C.L., 2003. Preventing violence and harassment in the workplace. European Foundation for the Improvement of Living and Working Conditions.

Mongin S.J., 2001. Adjustment for Non-Response in the Minnesota Nurses Study. Minneapolis, MN: Division of Environmental and Occupational Health, University of Minnesota. Retrieved November 21, 2010, from http://enhs.umn.edu/research/pdfs/response.adjustment.1.pdf.

Nolle, K.L., Guerino, P., Dinkes, R., 2007. Crime, Violence, Discipline, and Safety in U.S. Public Schools: Findings From the School Survey on Crime and Safety: 2005-06 (NCES 2007-361). National Center for Education Statistics, Institute of Education Sciences, U.S. Department of Education. Washington, DC.

Peek-Asa C., Howard J. Vargas, L., Kraus J.F., 1997. Incidence of Non-Fatal Workplace Assault Injuries Determined From Employer's Reports in California. Journal of Occupational and Environmental Medicine 39(1):44-50 
Peek-Asa C., Schaffer K.B., Kraus J.F., Howard J., 1998. Surveillance of Nonfatal Workplace Assault Injuries, Using Police and Employers' Reports. Journal of Occupational and Environmental Medicine 40(8):707-713

Rosenberg M., Fenley M., 1991. Violence in America: A Public Health Approach. Oxford University Press, New York.

Sage S.K., Gerberich S.G., Rayan A.D., Nachreiner N.M., Church T.R., Alexander B.H., Mongin S.J., 2010. School resources, resource allocation, and risk of physical assault against Minnesota educators. Accident Analysis and Prevention $42(1): 1-9$. 


\section{FIGURES and TABLES}


Figure1. Minnesota Educators' Study- Phase 1: Response Numbers

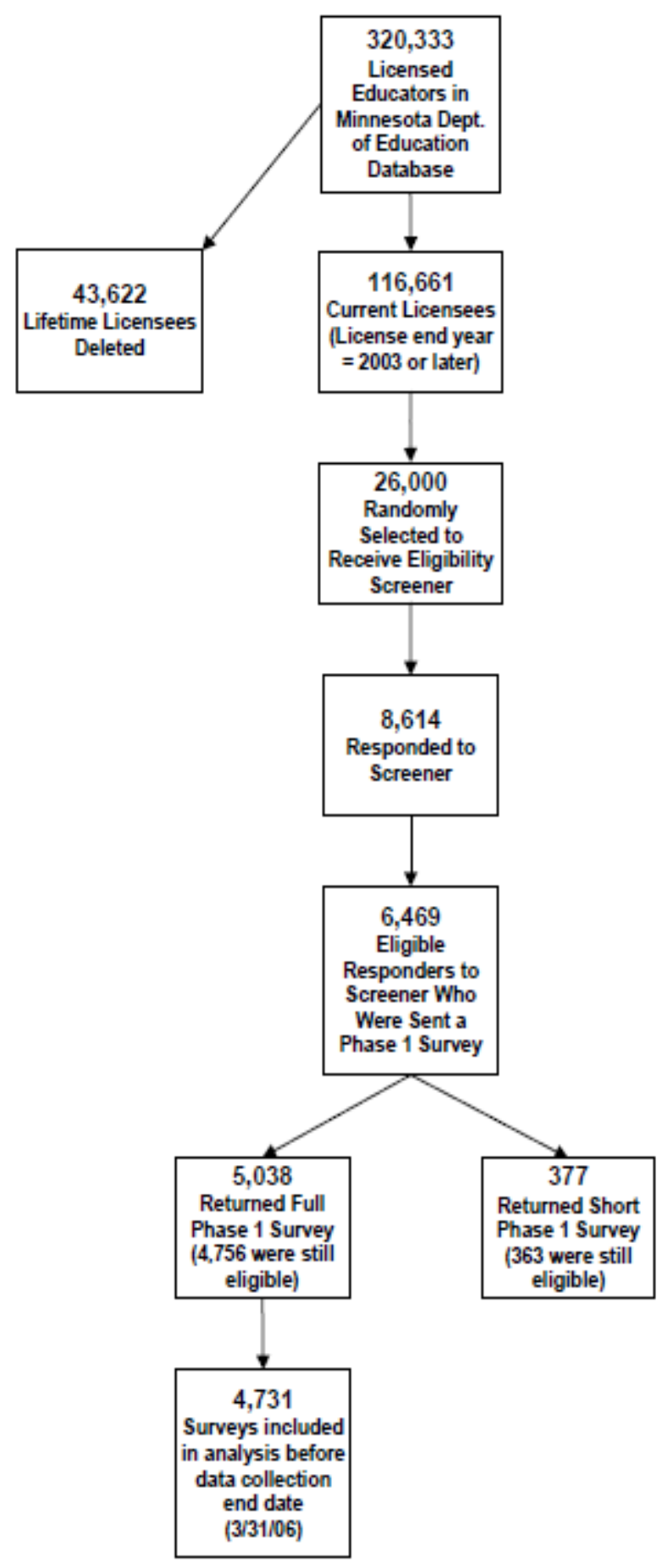


Figure2. Directed Acyclic Graph Example

Exposure of Interest: Grade Taught

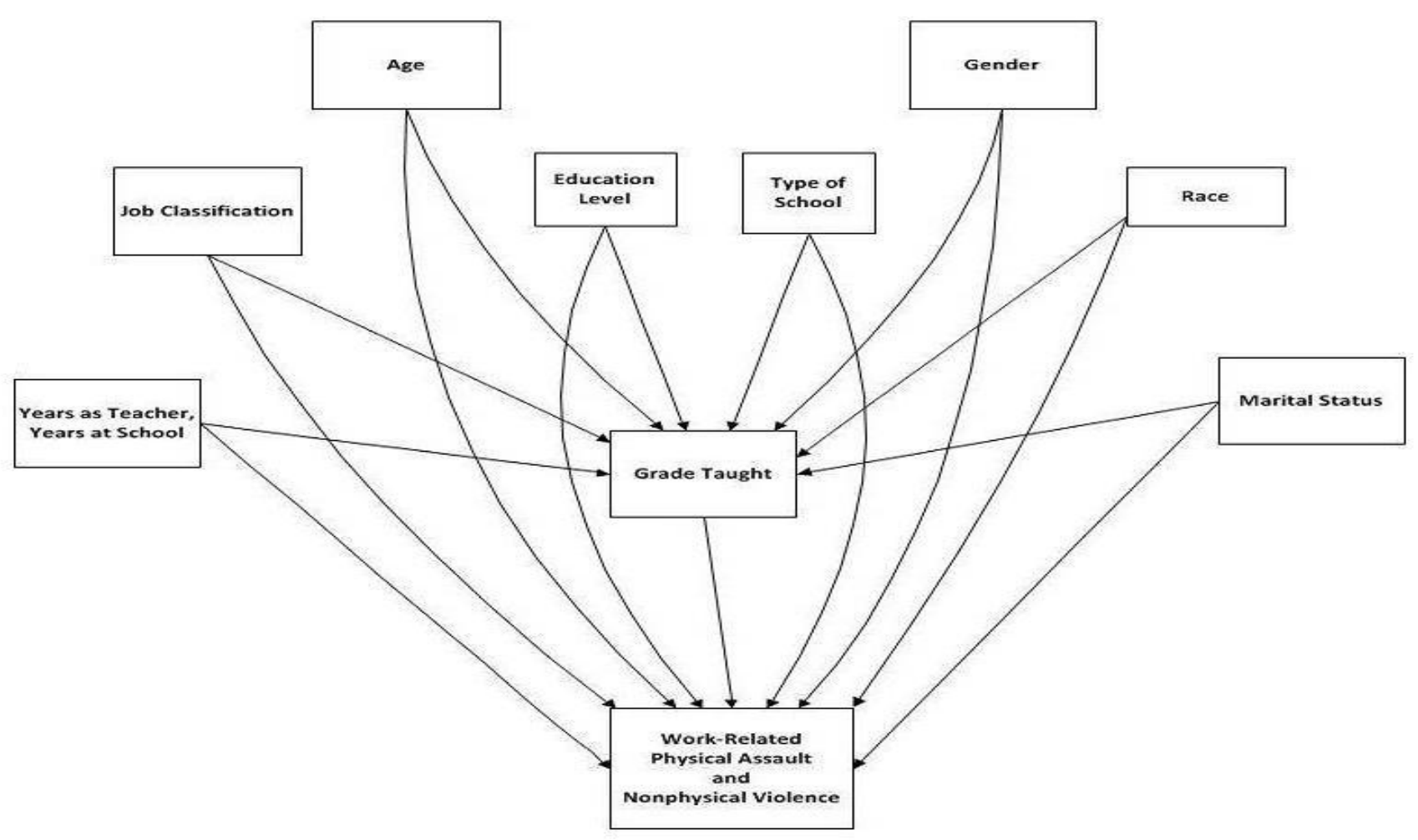


Table 1a. Physical Assault and Non-Physical Violence Rates Per 100,000 Working Hours Per Year: Violence Against Educators Study

\begin{tabular}{|c|c|c|c|c|c|c|c|c|}
\hline \multirow{3}{*}{ Variable } & \multicolumn{4}{|c|}{ Physical Assault } & \multicolumn{4}{|c|}{ Nonphysical Violence } \\
\hline & Number & Number & Injury & & Number & Number & Injury & \\
\hline & Responses & Events* & Rate & $95 \%$ C.I. & Responses & Events* & Rate & $95 \%$ C.1. \\
\hline Total Population & 4725 & 372 & 5.31 & $4.79-5.88$ & 4720 & 1834 & 26.37 & $25.18-27.61$ \\
\hline \multicolumn{9}{|l|}{ Gender } \\
\hline Male & 1102 & 68 & 4.22 & 3.35-5.31 & 1102 & 399 & 23.37 & $21.19-25.77$ \\
\hline Female & 3623 & 304 & 5.77 & $5.15-6.47$ & 3618 & 1435 & 27.64 & $26.24-29.11$ \\
\hline \multicolumn{9}{|l|}{ Age group } \\
\hline $20-29$ & 415 & 31 & 5.15 & $3.63-7.30$ & 415 & 165 & 26.45 & $22.68-30.85$ \\
\hline $30-39$ & 1032 & 83 & 5.32 & $4.28-6.62$ & 1031 & 423 & 27.46 & $24.94-30.22$ \\
\hline $40-49$ & 1242 & 107 & 5.66 & $4.68-6.86$ & 1242 & 491 & 26.28 & $24.04-28.73$ \\
\hline $50-59$ & 1730 & 134 & 5.09 & $4.29-6.03$ & 1726 & 654 & 24.91 & $23.07-26.90$ \\
\hline $60-79$ & 306 & 17 & 5.24 & $3.25-8.46$ & 306 & 101 & 31.28 & $25.70-38.06$ \\
\hline \multicolumn{9}{|l|}{ Ethnic } \\
\hline Hispanic & 29 & 6 & 14.49 & $6.54-32.12$ & 28 & 14 & 37.00 & $22.19-61.70$ \\
\hline Non Hispanic & 4661 & 361 & 5.22 & $4.71-5.79$ & 4659 & 1797 & 26.14 & $24.95-27.38$ \\
\hline \multicolumn{9}{|l|}{ Race } \\
\hline White & 4611 & 359 & 5.28 & $4.76-5.85$ & 4608 & 1775 & 26.16 & $24.96-27.41$ \\
\hline Non white & 75 & 7 & 5.29 & $2.40-11.67$ & 75 & 34 & 28.54 & $20.31-40.12$ \\
\hline \multicolumn{9}{|l|}{ Marital status } \\
\hline Married & 3749 & 271 & 4.92 & $4.37-5.55$ & 3747 & 1377 & 25.08 & 23.78-26.44 \\
\hline Other than married* & 942 & 93 & 6.40 & $5.20-7.88$ & 940 & 436 & 30.58 & $27.81-33.63$ \\
\hline \multicolumn{9}{|l|}{ Education } \\
\hline Associate + Bachelor's Degree & 1800 & 110 & 4.58 & 3.80-5.52 & 1799 & 616 & 25.90 & 23.95-28.01 \\
\hline Master & 2529 & 214 & 5.52 & $4.83-6.31$ & 2527 & 1038 & 36.50 & $24.93-28.17$ \\
\hline Education specialist degree & 309 & 36 & 6.65 & $4.77-9.29$ & 309 & 138 & 26.41 & $22.34-31.22$ \\
\hline Doctorate degree & 69 & 7 & 5.66 & $2.62-12.22$ & 65 & 28 & 24.60 & $16.97-35.66$ \\
\hline \multicolumn{9}{|l|}{ Years worked as a teacher } \\
\hline $0-9$ years & 1387 & 115 & 5.76 & $4.78-6.94$ & 1386 & 580 & 29.39 & 27.06-31.91 \\
\hline $10-19$ years & 1426 & 122 & 5.61 & $4.68-6.71$ & 1423 & 579 & 26.90 & $24.78-29.20$ \\
\hline 20-29 years & 1109 & 83 & 4.73 & $3.81-5.87$ & 1109 & 407 & 23.00 & $20.85-25.37$ \\
\hline$>30$ years & 778 & 47 & 4.05 & $3.04-5.39$ & 778 & 251 & 21.72 & $19.18-24.58$ \\
\hline \multicolumn{9}{|l|}{ Years worked in current school } \\
\hline $0-4$ years & 837 & 75 & 6.50 & $5.15-8.20$ & 834 & 357 & 32.49 & $29.27-36.05$ \\
\hline 5-9years & 587 & 40 & 4.53 & $3.30-6.21$ & 587 & 238 & 27.31 & $24.02-31.06$ \\
\hline $10-19$ years & 725 & 69 & 5.99 & $4.70-7.62$ & 723 & 284 & 25.41 & $22.60-28.57$ \\
\hline$>20$ & 536 & 17 & 2.09 & $1.30-3.35$ & 536 & 179 & 21.35 & $18.42-24.75$ \\
\hline
\end{tabular}


Table 1a. (continued) Physical Assault and Non-Physical Violence Rates Per 100,000 Working Hours Per Year: Violence Against Educators Study

\begin{tabular}{|c|c|c|c|c|c|c|c|c|}
\hline \multirow[b]{2}{*}{ Variable } & \multicolumn{4}{|c|}{ Physical Assault } & \multicolumn{4}{|c|}{ Nonphysical Violence } \\
\hline & $\begin{array}{r}\text { Number } \\
\text { Responses }\end{array}$ & $\begin{array}{l}\text { Number } \\
\text { Events* }\end{array}$ & $\begin{array}{r}\text { Injury } \\
\text { Rate }\end{array}$ & 95\% C.I. & $\begin{array}{r}\text { Number } \\
\text { Responses }\end{array}$ & $\begin{array}{l}\text { Number } \\
\text { Events* }\end{array}$ & $\begin{array}{r}\text { Injury } \\
\text { Rate }\end{array}$ & $95 \%$ C.I. \\
\hline \multicolumn{9}{|l|}{ Type of school } \\
\hline Public & 4004 & 327 & 5.53 & 4.96-6.17 & 3998 & 1553 & 26.38 & $25.10-27.73$ \\
\hline Public Alternative & 159 & 20 & 9.54 & $6.26-14.55$ & 159 & 113 & 50.63 & $42.15-60.80$ \\
\hline Public Charter & 86 & 6 & 3.32 & $1.30-8.47$ & 86 & 39 & 28.31 & $20.55-39.01$ \\
\hline Public Magnet & 64 & 8 & 8.08 & 4.07-16.01 & 64 & 34 & 33.24 & $23.72-46.58$ \\
\hline Private (Parochial) & 309 & 4 & 0.94 & $0.36-2.41$ & 309 & 60 & 13.13 & 10.19-16.91 \\
\hline Private(Non-Parochial) & 64 & 1 & 0.67 & $0.06-7.26$ & 64 & 15 & 15.89 & $9.74-25.92$ \\
\hline No one school type was most common & 28 & 6 & 17.18 & $7.65-38.55$ & 29 & 16 & 43.27 & $26.16-71.56$ \\
\hline \multicolumn{9}{|l|}{ Job classification } \\
\hline Full-time & 3764 & 333 & 5.33 & $4.79-5.94$ & 3759 & 1536 & 24.76 & $23.55-26.03$ \\
\hline Part-time & 420 & 18 & 4.35 & 2.74-6.92 & 420 & 138 & 34.49 & $29.25-40.66$ \\
\hline Othert & 523 & 21 & 6.32 & 4.09-9.77 & 523 & 158 & 47.75 & 40.76-55.95 \\
\hline \multicolumn{9}{|l|}{ Activity } \\
\hline Classroom teacher & 3193 & 148 & 3.22 & 2.74-3.78 & 3191 & 1152 & 24.66 & $23.27-26.13$ \\
\hline Administrator + Superintendent + Dean of students & 197 & 19 & 4.76 & $3.04-7.48$ & 196 & 89 & 22.69 & $18.45-27.91$ \\
\hline School psychologist & 46 & 7 & 11.02 & $5.35-22.71$ & 46 & 23 & 35.56 & $23.77-53.18$ \\
\hline School social worker & 66 & 15 & 16.14 & $9.84-26.48$ & 66 & 31 & 35.56 & $25.48-49.63$ \\
\hline Special education & 595 & 126 & 14.71 & $12.40-17.47$ & 594 & 308 & 34.91 & $31.23-39.03$ \\
\hline Department chair/ instructional leader & 22 & 5 & 14.58 & $5.96-35.66$ & 22 & 7 & 20.93 & $9.92-44.16$ \\
\hline $\begin{array}{l}\text { Teacher assistant/aide + advisor to extra- curricular } \\
\text { activities + other }\end{array}$ & 346 & 24 & 5.22 & $3.48-7.82$ & 344 & 124 & 28.03 & $23.53-33.40$ \\
\hline No one activity was most common & 250 & 28 & 7.60 & $5.20-11.10$ & 251 & 98 & 27.42 & $22.46-33.47$ \\
\hline \multicolumn{9}{|l|}{ Class size } \\
\hline$<10$ & 723 & 140 & 14.00 & $11.90-16.48$ & 721 & 342 & 33.61 & $30.25-37.34$ \\
\hline 10 to less than 25 & 1665 & 115 & 4.66 & $3.87-5.61$ & 1664 & 556 & 22.84 & $21.01-24.84$ \\
\hline$>=25$ & 1865 & 77 & 2.88 & 2.31-3.59 & 1864 & 755 & 27.36 & $25.48-29.39$ \\
\hline \multicolumn{9}{|l|}{ Grade } \\
\hline Kindergartern-2nd grade & 659 & 56 & 6.03 & $4.65-7.80$ & 659 & 161 & 17.13 & $14.70-19.97$ \\
\hline 3-6 grade & 908 & 60 & 4.53 & $3.53-5.83$ & 908 & 294 & 22.02 & $19.65-24.68$ \\
\hline 7-9 grade & 609 & 30 & 3.44 & 2.43-4.87 & 609 & 276 & 29.45 & $26.15-33.18$ \\
\hline $10-12$ grade & 426 & 14 & 2.09 & $1.24-3.52$ & 426 & 200 & 29.11 & $25.31-33.48$ \\
\hline No one grade level was most frequently taught & 1641 & 171 & 7.34 & $6.30-8.54$ & 1637 & 719 & 31.52 & $29.29-33.92$ \\
\hline
\end{tabular}


Table 1b. Non-Physical Violence Rates Per 100,000 Working Hours Per Year: Violence Against Educators Study

\begin{tabular}{|c|c|c|c|c|c|c|c|c|c|c|c|c|c|c|c|c|}
\hline \multirow[b]{2}{*}{ Variable } & \multicolumn{4}{|c|}{ Threat } & \multicolumn{4}{|c|}{ Sexual Harassment } & \multicolumn{4}{|c|}{ Verbal Abuse } & \multicolumn{4}{|c|}{ Bullying } \\
\hline & $\begin{array}{r}\text { Number } \\
\text { Responses }\end{array}$ & $\begin{array}{l}\text { Number } \\
\text { Events* }\end{array}$ & $\begin{array}{r}\text { Injury } \\
\text { Rate }\end{array}$ & 95\% C.I. & $\begin{array}{r}\text { Number } \\
\text { Responses }\end{array}$ & $\begin{array}{l}\text { Number } \\
\text { Events* }\end{array}$ & $\begin{array}{r}\text { Injury } \\
\text { Rate }\end{array}$ & 95\% C.I. & $\begin{array}{r}\text { Response } \\
\mathrm{s} \\
\end{array}$ & $\begin{array}{l}\text { Number } \\
\text { Events* }\end{array}$ & $\begin{array}{r}\text { Injury } \\
\text { Rate }\end{array}$ & 95\% C.I. & $\begin{array}{r}\text { Number } \\
\text { Responses }\end{array}$ & $\begin{array}{l}\text { Number } \\
\text { Events* }\end{array}$ & $\begin{array}{r}\text { Injury } \\
\text { Rate }\end{array}$ & 95\% C.I. \\
\hline Total Population & 4719 & 957 & 34.82 & $32.70-37.08$ & 4717 & 212 & 7.58 & $6.63-8.68$ & 4720 & 1576 & 55.48 & $52.79-58.31$ & 4719 & 550 & 19.62 & $18.04-21.33$ \\
\hline \multicolumn{17}{|l|}{ Gender } \\
\hline Male & 1102 & 219 & 35.7 & $31.40-40.59$ & 1102 & 38 & 5.73 & $4.16-7.89$ & 1102 & 348 & 52.87 & $47.57-58.75$ & 1102 & 94 & 15.17 & $12.46-18.47$ \\
\hline Female & 3617 & 738 & 34.47 & $32.07-37.04$ & 3615 & 174 & 8.33 & 7.19-9.65 & 3618 & 1228 & 56.53 & $53.44-59.81$ & 3617 & 456 & 21.41 & $19.54-23.46$ \\
\hline \multicolumn{17}{|l|}{ Age group } \\
\hline $20-29$ & 415 & 95 & 37.92 & $31.06-46.29$ & 415 & 26 & 9.46 & $6.34-14.10$ & 415 & 134 & 52.02 & $43.88-61.68$ & 415 & 61 & 23.67 & $18.39-30.47$ \\
\hline 30-39 & 1031 & 240 & 38.06 & $33.54-43.19$ & 1031 & 53 & 8.1 & $6.16-10.65$ & 1031 & 363 & 56.08 & $50.53-62.23$ & 1031 & 124 & 19.23 & $16.09-22.97$ \\
\hline $40-49$ & 1242 & 246 & 32.81 & $28.95-37.18$ & 1242 & 66 & 8.57 & $6.71-10.94$ & 1242 & 412 & 53.91 & $48.90-59.43$ & 1242 & 141 & 18.61 & 15.76-21.97 \\
\hline 50-59 & 1725 & 323 & 31.33 & $28.07-34.96$ & 1732 & 56 & 5.33 & $4.09-6.96$ & 1726 & 579 & 56.22 & $51.81-61.02$ & 1725 & 195 & 18.64 & 16.17-21.49 \\
\hline $60-79$ & 306 & 53 & 41.82 & $31.80-54.99$ & 306 & 11 & 8.07 & $4.33-15.05$ & 306 & 88 & 69.86 & $56.52-86.35$ & 306 & 29 & 22.14 & 15.19-32.26 \\
\hline \multicolumn{17}{|l|}{ Ethnic } \\
\hline Hispanic & 28 & 6 & 27.6 & $12.46-61.14$ & 28 & 1 & 3.66 & $0.41-32.51$ & 28 & 12 & 57.01 & $32.78-99.14$ & 28 & 4 & 17.88 & $6.66-48.02$ \\
\hline Non Hispanic & 4658 & 942 & 34.89 & $32.75-37.18$ & 4656 & 210 & 7.68 & $6.71-8.80$ & 4659 & 1542 & 55.32 & $52.61-58.18$ & 4658 & 538 & 19.6 & 18.01-21.33 \\
\hline \multicolumn{17}{|l|}{ Race } \\
\hline White & 4607 & 928 & 34.82 & $32.67-37.12$ & 4605 & 204 & 7.6 & $6.63-8.71$ & 4608 & 1524 & 55.34 & $52.61-58.22$ & 4607 & 532 & 19.56 & $17.97-21.30$ \\
\hline Non white & 75 & 17 & 34.6 & 21.73-55.09 & 75 & 6 & 9.37 & $3.83-22.90$ & 75 & 29 & 56.76 & $39.48-81.62$ & 75 & 11 & 24.54 & $14.12-42.63$ \\
\hline \multicolumn{17}{|l|}{ Marital status } \\
\hline Married & 3747 & 727 & 35.33 & $32.88-37.96$ & 3745 & 141 & 6.57 & $5.56-7.76$ & 3747 & 1169 & 54.7 & $51.64-57.95$ & 3746 & 409 & 19.15 & $17.37-21.11$ \\
\hline Other than married* & 940 & 222 & 33.47 & $29.31-38.22$ & 940 & 70 & 10.91 & $8.65-13.76$ & 940 & 388 & 57.62 & $52.08-63.75$ & 940 & 135 & 21.13 & $17.88-24.96$ \\
\hline \multicolumn{17}{|l|}{ Education } \\
\hline Associate + Bachelor's Degree & 1798 & 304 & 35.65 & $31.93-39.80$ & 1798 & 70 & 7.86 & 6.22-9.94 & 1799 & 533 & 60.33 & $55.44-65.66$ & 1799 & 184 & 20.93 & $18.13-24.17$ \\
\hline Master & 2527 & 551 & 34.34 & $31.61-37.31$ & 2525 & 113 & 7.12 & $5.94-8.55$ & 2527 & 892 & 53.68 & $50.23-57.36$ & 2526 & 310 & 18.95 & 16.94-21.18 \\
\hline Education specialist degree & 309 & 81 & 35.71 & 28.81-44.27 & 309 & 27 & 11.02 & $7.49-16.22$ & 309 & 115 & 47.93 & $39.82-57.70$ & 309 & 46 & 20.35 & $15.31-27.05$ \\
\hline Doctorate degree & 68 & 17 & 35.62 & $22.14-57.33$ & 68 & 2 & 4.52 & $1.19-17.18$ & 68 & 23 & 48.42 & $32.20-72.82$ & 68 & 6 & 11.56 & $5.02-26.65$ \\
\hline \multicolumn{17}{|l|}{ Years worked as a teacher } \\
\hline 0-9 years & 1386 & 320 & 38.52 & $34.51-42.99$ & 1386 & 86 & 9.86 & $7.94-12.25$ & 1386 & 481 & 56.69 & $51.79-62.06$ & 1386 & 185 & 22.05 & $19.07-25.49$ \\
\hline $10-19$ years & 1423 & 302 & 33.83 & $30.23-37.85$ & 1422 & 55 & 5.95 & $4.55-7.78$ & 1423 & 499 & 53.95 & $49.35-58.97$ & 1422 & 153 & 16.85 & $14.37-19.76$ \\
\hline 20-29 years & 1109 & 201 & 30.62 & $26.65-35.19$ & 1109 & 45 & 6.72 & 4.99-9.04 & 1109 & 357 & 53.8 & $48.45-59.75$ & 1109 & 124 & 18.56 & $15.53-22.19$ \\
\hline$>30$ years & 777 & 129 & 33.48 & $28.15-39.82$ & 776 & 26 & 6.62 & 4.48-9.79 & 778 & 223 & 57.88 & $50.74-66.02$ & 778 & 84 & 21.41 & $17.25-26.59$ \\
\hline \multicolumn{17}{|l|}{ Years worked in current school } \\
\hline $0-4$ years & 834 & 201 & 41.05 & $35.82-47.04$ & 834 & 43 & 8.79 & $6.55-11.80$ & 834 & 306 & 59.62 & $53.24-66.75$ & 834 & 113 & 22.3 & $18.53-26.82$ \\
\hline 5-9years & 587 & 118 & 34.56 & 28.89-41.34 & 586 & 25 & 6.71 & $4.47-10.09$ & 587 & 204 & 56.73 & $49.33-65.24$ & 586 & 67 & 19.53 & $15.39-24.80$ \\
\hline $10-19$ years & 723 & 158 & 35.29 & $30.14-41.33$ & 722 & 30 & 6.87 & 4.80-9.83 & 723 & 244 & 54.87 & $48.34-62.27$ & 723 & 84 & 18.52 & $14.89-23.02$ \\
\hline$>20$ & 535 & 84 & 30.23 & $24.38-37.47$ & 535 & 18 & 6.07 & $3.76-9.81$ & 536 & 159 & 56.43 & $48.24-66.01$ & 536 & 52 & 18.23 & $13.83-24.02$ \\
\hline
\end{tabular}


Table 1b. (continued) Non-Physical Violence Rates Per 100,000 Working Hours Per Year: Violence Against Educators Study

\begin{tabular}{|c|c|c|c|c|c|c|c|c|c|c|c|c|c|c|c|c|}
\hline \multirow[b]{3}{*}{ Variable } & \multicolumn{4}{|c|}{ Threat } & \multicolumn{4}{|c|}{ Sexual Harassment } & \multicolumn{4}{|c|}{ Verbal Abuse } & \multicolumn{4}{|c|}{ Bullying } \\
\hline & Number & Number & Injury & & Number & Number & Injury & & $\begin{array}{l}\text { Number } \\
\text { Response }\end{array}$ & Number & Injury & & Number & Number & Injury & \\
\hline & Responses & Events* & Rate & 95\% C.I. & Responses & Events* & Rate & 95\% C.I. & s & Events* & Rate & 95\% C.I. & . Responses & Events* & Rate & 95\% C.I. \\
\hline \multicolumn{17}{|l|}{ Type of school } \\
\hline Public & 3997 & 787 & 33.73 & $31.47-36.16$ & 3995 & 167 & 7.14 & $6.14-8.30$ & 3998 & 1330 & 55.28 & $52.36-58.36$ & 3997 & 456 & 18.99 & $17.31-20.83$ \\
\hline Public Alternative & 159 & 75 & 46.84 & $37.62-58.33$ & 159 & 25 & 13.89 & $9.28-20.78$ & 159 & 109 & 65.04 & 53.99-78.35 & 159 & 36 & 23.39 & $17.15-31.90$ \\
\hline Public Charter & 86 & 25 & 39.24 & $26.33-58.49$ & 86 & 4 & 6.84 & $2.63-17.79$ & 86 & 34 & 53.86 & $38.31-75.72$ & 86 & 15 & 25.72 & 15.71-42.11 \\
\hline Public Magnet & 64 & 25 & 45.75 & $30.84-67.88$ & 64 & 5 & 10.03 & $4.32-23.29$ & 64 & 30 & 55.74 & $38.99-79.68$ & 64 & 7 & 12.5 & $5.88-26.58$ \\
\hline Private (Parochial) & 309 & 23 & 26.33 & $17.71-39.14$ & 309 & 4 & 4.24 & $1.58-11.38$ & 309 & 40 & 42.2 & $30.86-57.72$ & 309 & 23 & 25.79 & $17.28-38.49$ \\
\hline Private(Non-Parochial) & 64 & 10 & 40.13 & $22.26-72.33$ & 64 & 1 & 2.36 & $0.21-26.78$ & 64 & 13 & 43.91 & 25.01-77.12 & 64 & 7 & 24.75 & $11.69-52.39$ \\
\hline No one school type was most & 29 & 11 & 47.28 & 25.43-87.91 & 29 & 5 & 21.34 & $8.48-53.72$ & 29 & 16 & 72 & 43.56-119.00 & 29 & 5 & 18.99 & 7.14-50.52 \\
\hline \multicolumn{17}{|l|}{$\begin{array}{l}\text { common } \\
\text { Job classification }\end{array}$} \\
\hline Full-time & 3758 & 821 & 32.99 & $30.83-35.31$ & 3756 & 184 & 7.22 & $6.25-8.35$ & 3759 & 1322 & 51.5 & $48.78-54.38$ & 3758 & 469 & 18.51 & $16.91-20.27$ \\
\hline Part-time & 420 & 64 & 45.04 & $35.60-56.97$ & 420 & 11 & 7.25 & 4.04-13.03 & 420 & 119 & 79.04 & 66.19-94.39 & 420 & 35 & 24.4 & $17.73-33.58$ \\
\hline Othert & 523 & 71 & 59.57 & $47.23-75.14$ & 523 & 16 & 14.51 & $9.06-23.23$ & 523 & 133 & 107.49 & $90.43-127.77$ & 523 & 45 & 35.86 & $26.59-48.37$ \\
\hline \multicolumn{17}{|l|}{ Activity } \\
\hline Classroom teacher & 3190 & 557 & 32.77 & $30.19-35.57$ & 3189 & 125 & 7.25 & $6.09-8.63$ & 3191 & 984 & 55.45 & $52.06-59.06$ & 3191 & 346 & 19.5 & $17.54-21.69$ \\
\hline $\begin{array}{l}\text { Administrator + Superintendent } \\
\text { + Dean of students }\end{array}$ & 196 & 57 & 32.48 & $25.08-42.05$ & 196 & 9 & 4.88 & 2.51-9.51 & 196 & 74 & 41.06 & $32.63-51.68$ & 196 & 27 & 15.35 & $10.54-22.36$ \\
\hline School psychologist & 46 & 14 & 39.67 & $23.65-66.53$ & 46 & 2 & 7.65 & $2.36-24.83$ & 46 & 20 & 56.93 & $36.98-87.67$ & 46 & 11 & 34.1 & 19.53-59.56 \\
\hline School social worker & 66 & 17 & 39.11 & $25.32-60.41$ & 66 & 4 & 8.75 & $3.49-21.94$ & 66 & 25 & 54.45 & $37.66-78.71$ & 66 & 6 & 14.01 & $6.77-28.96$ \\
\hline Special education & 594 & 201 & 44.27 & $38.63-50.73$ & 593 & 37 & 8.07 & 5.86-11.11 & 594 & 274 & 58.95 & $52.39-66.34$ & 593 & 96 & 21.48 & $17.65-26.13$ \\
\hline $\begin{array}{l}\text { Department chair/instructional } \\
\text { leader }\end{array}$ & 22 & 7 & 69.11 & $32.78-145.72$ & 22 & 3 & 29.84 & 9.59-92.87 & 22 & 7 & 69.12 & $32.79-145.72$ & 22 & 3 & 28.44 & 8.89-90.96 \\
\hline $\begin{array}{l}\text { Teacher assistant/aide + advisor } \\
\text { to extra- curricular activities + } \\
\text { other }\end{array}$ & 344 & 54 & 31.18 & 23.98-40.55 & 344 & 17 & 9.74 & $6.09-15.59$ & 344 & 103 & 57.71 & $47.58-70.00$ & 344 & 30 & 17.64 & $12.44-25.01$ \\
\hline $\begin{array}{l}\text { No one activity was most } \\
\text { common }\end{array}$ & 251 & 49 & 33.69 & $25.47-44.57$ & 251 & 14 & 8.53 & $4.89-14.87$ & 251 & 87 & 57.82 & $46.71-71.59$ & 251 & 30 & 19.89 & $13.82-28.63$ \\
\hline \multicolumn{17}{|l|}{ Class size } \\
\hline$<10$ & 721 & 212 & 43.15 & $37.82-49.24$ & 720 & 47 & 9.47 & $7.15-12.56$ & 721 & 304 & 59.93 & 53.59-67.03 & 720 & 118 & 24.12 & $20.21-28.78$ \\
\hline 10 to less than 25 & 1663 & 290 & 35.09 & $31.27-39.38$ & 1663 & 59 & 6.89 & $5.32-8.94$ & 1664 & 473 & 55.59 & $50.73-60.91$ & 1664 & 181 & 21.47 & $18.53-24.88$ \\
\hline$>=25$ & 1864 & 357 & 31.64 & $28.57-35.05$ & 1863 & 89 & 7.71 & $6.27-9.48$ & 1864 & 645 & 54.79 & $50.70-59.21$ & 1864 & 205 & 17.2 & 14.97-19.75 \\
\hline \multicolumn{17}{|l|}{ Grade } \\
\hline Kindergartern-2nd grade & 659 & 82 & 34.26 & $27.80-42.30$ & 659 & 11 & 4.15 & $2.27-7.59$ & 659 & 128 & 50.27 & 42.27-59.79 & 659 & 58 & 23.71 & $18.42-30.52$ \\
\hline $3-6$ grade & 907 & 156 & 34.22 & 29.30-39.97 & 907 & 25 & 6.24 & $4.34-8.98$ & 908 & 239 & 51.39 & $45.28-58.32$ & 908 & 97 & 20.9 & $17.14-25.49$ \\
\hline 7-9 grade & 609 & 140 & 33.2 & 28.15-39.15 & 607 & 38 & 8.66 & $6.26-11.97$ & 609 & 239 & 54.85 & $48.24-62.36$ & 608 & 104 & 24.51 & $20.22-29.71$ \\
\hline 10-12 grade & 426 & 106 & 34.2 & $28.27-41.38$ & 426 & 24 & 7.85 & $5.27-11.68$ & 426 & 181 & 57.09 & $49.26-66.16$ & 426 & 43 & 13.3 & 9.80-18.05 \\
\hline $\begin{array}{l}\text { No one grade level was most } \\
\text { frequently taught }\end{array}$ & 1637 & 375 & 36.67 & $33.16-40.55$ & 1637 & 97 & 9.03 & $7.37-11.06$ & 1637 & 631 & 59.79 & $55.26-64.69$ & 1637 & 203 & 18.72 & $16.26-21.54$ \\
\hline
\end{tabular}


Table 2a. Rate Ratios for Physical Assault and Non-Physical Violence Rates Per 100,000 Working Hours Per Year: Violence Against Educators Study

\begin{tabular}{|c|c|c|c|c|c|c|c|c|}
\hline \multirow[b]{3}{*}{ Variable } & \multicolumn{4}{|c|}{ Physical Assaults } & \multicolumn{4}{|c|}{ Nonphysical Violence } \\
\hline & \multirow{2}{*}{$\begin{array}{r}\text { Number } \\
\text { Responses }\end{array}$} & \multicolumn{2}{|c|}{ Number Estimate } & \multicolumn{2}{|r|}{ Number } & \multicolumn{2}{|c|}{ Number Estimate } & \multirow[b]{2}{*}{ 95\% C.I. } \\
\hline & & Events* & RR & 95\% C.I. & Responses & Events* & RR & \\
\hline \multicolumn{9}{|l|}{ Genderł } \\
\hline Male & 1103 & 68 & 0.73 & $0.56-0.94$ & 1103 & 399 & 0.85 & $0.76-0.94$ \\
\hline Female & 3628 & 304 & 1.00 & & 3628 & 1435 & 1.00 & \\
\hline \multicolumn{9}{|l|}{ Age group $\neq$} \\
\hline $20-29$ & 416 & 31 & 1.01 & $0.69-1.49$ & 416 & 165 & 1.06 & $0.89-1.26$ \\
\hline 30-39 & 1033 & 83 & 1.05 & $0.79-1.38$ & 1033 & 423 & 1.10 & $0.97-1.25$ \\
\hline $40-49$ & 1242 & 107 & 1.11 & $0.86-1.44$ & 1242 & 491 & 1.05 & $0.94-1.19$ \\
\hline 50-59 & 1732 & 134 & 1.00 & - & 1732 & 654 & 1.00 & \\
\hline $60-79$ & 308 & 17 & 1.03 & $0.62-1.71$ & 308 & 101 & 1.26 & $1.02-1.55$ \\
\hline \multicolumn{9}{|l|}{ Raceł } \\
\hline White & 4613 & 359 & 1.00 & - & 4613 & 1775 & 1.00 & \\
\hline Non white & 75 & 7 & 1.00 & $0.45-2.23$ & 75 & 34 & 1.09 & $0.77-1.54$ \\
\hline \multicolumn{9}{|l|}{ Marital status§ } \\
\hline Married & 3740 & 271 & 1.00 & - & 3740 & 1373 & 1.00 & \\
\hline Other than married ${ }^{\dagger}$ & 935 & 92 & 1.28 & $1.00-1.64$ & 935 & 431 & 1.20 & $1.07-1.34$ \\
\hline \multicolumn{9}{|l|}{ Education** } \\
\hline Associate + Bachelor's Degree & 1781 & 108 & 1.00 & - & 1781 & 607 & 1.00 & \\
\hline Master & 2516 & 213 & 1.23 & $0.97-1.56$ & 2516 & 1032 & 1.04 & $0.94-1.15$ \\
\hline Education specialist degree & 307 & 35 & 1.53 & $1.03-2.26$ & 307 & 136 & 1.05 & $0.87-1.27$ \\
\hline Doctorate degree & 68 & 7 & 1.38 & $0.62-3.09$ & 68 & 28 & 0.98 & $0.67-1.45$ \\
\hline \multicolumn{9}{|l|}{ Years worked as a teachertt } \\
\hline $0-10$ years & 1371 & 114 & 1.00 & & 1371 & 572 & 1.00 & \\
\hline $10-19$ years & 1414 & 121 & 0.85 & $0.63-1.15$ & 1414 & 573 & 0.83 & $0.72-0.95$ \\
\hline 20-29 years & 1103 & 82 & 0.66 & $0.45-0.95$ & 1103 & 405 & 0.66 & $0.56-0.78$ \\
\hline$>30$ years & 774 & 46 & 0.55 & $0.35-0.86$ & 774 & 249 & 0.60 & $0.49-0.73$ \\
\hline \multicolumn{9}{|c|}{ Years worked in current school $\ddagger$} \\
\hline $0-4$ years & 823 & 71 & 1.00 & - & 823 & 348 & 1.00 & \\
\hline 5-9years & 575 & 40 & 0.76 & $0.50-1.14$ & 575 & 233 & 0.91 & $0.76-1.08$ \\
\hline $10-19$ years & 719 & 69 & 1.08 & $0.71-1.63$ & 719 & 284 & 0.90 & $0.74-1.08$ \\
\hline$>20$ & 528 & 17 & 0.39 & $0.21-0.73$ & 528 & 175 & 0.80 & $0.63-1.01$ \\
\hline \multicolumn{9}{|c|}{ *The number of events was established per educator } \\
\hline \multirow{2}{*}{\multicolumn{9}{|c|}{$\begin{array}{l}\text { † Other than married: living as married. Living with a domestic partner, never married, separated, divorced, widowed } \\
\ddagger \text { Adjusted for non-response }\end{array}$}} \\
\hline & & & & & & & & \\
\hline \multicolumn{9}{|c|}{$\S$ Adjusted for gender, race and age } \\
\hline \multicolumn{9}{|c|}{${ }^{* *}$ Adjusted for gender, race, age and marry status } \\
\hline \multicolumn{9}{|c|}{ †† Adjusted for gender, race, age, marry status and education } \\
\hline 㧊 Adjusted for gender, race, a & education, jo & $\mathrm{bc}$ & & & & & & \\
\hline
\end{tabular}


Table 2a. (continued) Rate Ratios for Physical Assault and Non-Physical Violence Rates Per 100,000 Working Hours Per Year: Violence Against Educators Study

\begin{tabular}{|c|c|c|c|c|c|c|c|c|}
\hline \multirow[b]{3}{*}{ Variable } & \multicolumn{4}{|c|}{ Physical Assaults } & \multicolumn{4}{|c|}{ Nonphysical Violence } \\
\hline & \multirow{2}{*}{$\begin{array}{r}\text { Number } \\
\text { Responses }\end{array}$} & \multirow{2}{*}{\multicolumn{2}{|c|}{$\begin{array}{cr}\text { Number } & \text { Estimate } \\
\text { Events* } & \text { RR }\end{array}$}} & \multirow[b]{2}{*}{ 95\% C.I. } & \multirow{2}{*}{$\begin{array}{r}\text { Number } \\
\text { Responses }\end{array}$} & \multicolumn{2}{|c|}{ Number Estimate } & \multirow[b]{2}{*}{ 95\% C.I. } \\
\hline & & & & & & Events* & RR & \\
\hline \multicolumn{9}{|l|}{ Type of school¥ } \\
\hline Public & 3937 & 319 & 1.00 & - & 3935 & 1520 & 1.00 & - \\
\hline Public Alternative & 157 & 20 & 1.73 & $1.11-2.68$ & 157 & 112 & 1.93 & $1.59-2.34$ \\
\hline Public Charter & 85 & 6 & 0.58 & $0.22-1.49$ & 85 & 39 & 1.04 & $0.75-1.45$ \\
\hline Public Magnet & 63 & 7 & 1.24 & $0.60-2.58$ & 63 & 33 & 1.20 & $0.85-1.71$ \\
\hline Private (Parochial) & 309 & 4 & 0.17 & $0.06-0.44$ & 309 & 60 & 0.51 & $0.39-0.66$ \\
\hline Private(Non-Parochial) & 64 & 1 & 0.13 & $0.01-1.38$ & 64 & 15 & 0.62 & $0.38-1.02$ \\
\hline No one school type was most common & 29 & 6 & 3.05 & $1.33-6.96$ & 29 & 16 & 1.38 & $0.83-2.29$ \\
\hline \multicolumn{9}{|l|}{ Job classification§ } \\
\hline Full-time & 2071 & 174 & 1.00 & - & 1914 & 773 & 1.00 & - \\
\hline Part-time & 249 & 12 & 0.78 & $0.42-1.43$ & 205 & 75 & 1.44 & $1.13-1.82$ \\
\hline Othert & 343 & 11 & 0.70 & $0.36-1.36$ & 308 & 97 & 1.73 & $1.36-2.19$ \\
\hline \multicolumn{9}{|l|}{ Activity** } \\
\hline Classroom teacher & 1837 & 78 & 1.00 & - & 1837 & 654 & 1.00 & \\
\hline $\begin{array}{l}\text { Administrator + Superintendent }+ \text { Dean of } \\
\text { students }\end{array}$ & 71 & 9 & 1.81 & $0.82-3.98$ & 71 & 38 & 1.09 & $0.75-1.58$ \\
\hline School psychologist & 18 & 1 & 1.03 & $0.16-6.52$ & 18 & 10 & 1.33 & $0.69-2.56$ \\
\hline School social worker & 33 & 7 & 4.50 & $2.07-9.76$ & 33 & 13 & 1.14 & $0.66-1.95$ \\
\hline Special education & 348 & 69 & 4.39 & $3.13-6.16$ & 348 & 183 & 1.45 & $1.23-1.72$ \\
\hline Department chair/instructional leader & 11 & 2 & 3.01 & $0.70-12.88$ & 11 & 2 & 0.49 & $0.13-1.88$ \\
\hline $\begin{array}{l}\text { Teacher assistant/aide + advisor to extra- } \\
\text { curricular activities + other }\end{array}$ & 194 & 11 & 1.35 & $0.70-2.59$ & 194 & 74 & 1.13 & $0.88-1.44$ \\
\hline No one activity was most common & 131 & 20 & 4.01 & $2.42-6.63$ & 131 & 64 & 1.41 & $1.09-1.83$ \\
\hline \multicolumn{9}{|l|}{ Class sizett } \\
\hline$<10$ & 411 & 79 & 2.71 & $1.92-3.82$ & 411 & 205 & 1.43 & $1.20-1.71$ \\
\hline 10 to less than 25 & 952 & 59 & 1.00 & - & 952 & 317 & 1.00 & 1 \\
\hline$>=25$ & 1069 & 44 & 0.54 & $0.36-0.81$ & 1069 & 427 & 1.16 & $1.00-1.35$ \\
\hline \multicolumn{9}{|l|}{ Grade§§ } \\
\hline Kindergartern-2nd grade & 371 & 28 & 1.00 & - & 371 & 91 & 1.00 & \\
\hline 3-6 grade & 530 & 32 & 0.78 & $0.46-1.31$ & 530 & 169 & 1.30 & $1.00-1.68$ \\
\hline $7-9$ grade & 319 & 17 & 0.72 & $0.39-1.33$ & 319 & 146 & 1.79 & $1.36-2.34$ \\
\hline $10-12$ grade & 219 & 6 & 0.39 & $0.17-0.91$ & 219 & 103 & 1.76 & $1.32-2.35$ \\
\hline No one grade level was most frequently taught & 982 & 98 & 1.36 & $0.87-2.11$ & 982 & 435 & 1.77 & $1.41-2.24$ \\
\hline \multicolumn{9}{|c|}{ * The number of events was established per educator } \\
\hline \multicolumn{9}{|c|}{ † Other: Long-call substitute, building substitute, all other substitute } \\
\hline \multicolumn{9}{|c|}{ ‡ Adjusted for gender, race, age, merry status, education, jobclass, year as a teacher } \\
\hline \multicolumn{9}{|c|}{$\S$ Adjusted for gender, race, age, merry status, education, school type, grade and year worked } \\
\hline \multicolumn{9}{|c|}{ ** Adjusted for gender, race, age, merry status, education, jobclass, year as a teacher, year worked and school type } \\
\hline \multicolumn{9}{|c|}{ †† Adjusted for gender, race, age, education, jobclass, year as a teacher, year worked and school type and activity } \\
\hline$\S \S$ Adjusted for gender, race, age, merry status, & education, jo & obclass, ye & ar as a tea & cher, year wc & orked, schoo & I type and & & \\
\hline
\end{tabular}


Table 2b. Rate Ratios for Non-Physical Violence Rates Per 100,000 Working Hours Per Year: Violence Against Educators Study

\begin{tabular}{|c|c|c|c|c|c|c|c|c|c|c|c|c|c|c|c|c|}
\hline \multirow[b]{3}{*}{ Variable } & \multicolumn{4}{|c|}{ Threat } & \multicolumn{4}{|c|}{ Sexual harassment } & \multicolumn{4}{|c|}{ Verbal abuse } & \multicolumn{4}{|c|}{ Bullying } \\
\hline & \multirow{2}{*}{$\begin{array}{r}\text { Number } \\
\text { Responses }\end{array}$} & \multicolumn{2}{|c|}{ Number Estimate } & \multirow{2}{*}{\multicolumn{2}{|c|}{$\begin{array}{r}\text { Number } \\
95 \% \text { C.I. Responses }\end{array}$}} & \multirow{2}{*}{$\begin{array}{l}\text { Number } \\
\text { Events* }\end{array}$} & \multirow{2}{*}{$\begin{array}{r}\text { Estimate } \\
\mathrm{RR}\end{array}$} & \multirow{2}{*}{$95 \%$ C.I. } & \multirow{2}{*}{$\begin{array}{r}\text { Number } \\
\text { Responses }\end{array}$} & \multirow{2}{*}{$\begin{array}{l}\text { Number } \\
\text { Events* }\end{array}$} & \multirow{2}{*}{$\begin{array}{r}\text { Estimate } \\
\text { RR }\end{array}$} & \multirow{2}{*}{$95 \%$ C.I. } & \multirow{2}{*}{$\begin{array}{r}\text { Number } \\
\text { Responses }\end{array}$} & \multirow{2}{*}{$\begin{array}{l}\text { Number } \\
\text { Events* }\end{array}$} & \multirow{2}{*}{$\begin{array}{r}\text { Estimate } \\
\mathrm{RR}\end{array}$} & \multirow[b]{2}{*}{$95 \%$ C.I. } \\
\hline & & Events* & $\mathrm{RR}$ & & & & & & & & & & & & & \\
\hline \multicolumn{17}{|c|}{ - } \\
\hline Male & 1103 & 219 & 1.04 & $0.89-1.20$ & 1103 & 38 & 0.69 & $0.48-0.98$ & 1103 & 348 & 0.94 & $0.83-1.05$ & 1103 & 94 & 0.71 & $0.57-0.88$ \\
\hline Female & 3628 & 738 & 1.00 & & 3628 & 174 & 1.00 & - & 3628 & 1228 & 1.00 & - & 3628 & 456 & 1.00 & \\
\hline \multicolumn{17}{|l|}{ Age group $\neq$} \\
\hline $20-29$ & 416 & 95 & 1.21 & $0.96-1.52$ & 416 & 26 & 1.77 & $1.10-2.86$ & 416 & 134 & 0.93 & $0.77-1.12$ & 416 & 61 & 1.27 & $0.95-1.70$ \\
\hline 30-39 & 1033 & 240 & 1.21 & $1.03-1.44$ & 1033 & 53 & 1.52 & $1.04-2.22$ & 1033 & 363 & 1.00 & $0.87-1.14$ & 1033 & 124 & 1.03 & $0.82-1.29$ \\
\hline $40-49$ & 1242 & 246 & 1.05 & $0.89-1.24$ & 1242 & 66 & 1.61 & $1.12-2.31$ & 1242 & 412 & 0.96 & $0.84-1.09$ & 1242 & 141 & 1.00 & $0.80-1.24$ \\
\hline $50-59$ & 1732 & 323 & 1.00 & - & 1732 & 56 & 1.00 & - & 1732 & 579 & 1.00 & - & 1732 & 195 & 1.00 & \\
\hline 60-79 & 308 & 53 & 1.34 & $0.99-1.79$ & 308 & 11 & 1.51 & $0.77-2.98$ & 308 & 88 & 1.24 & $0.99-1.56$ & 308 & 29 & 1.19 & $0.79-1.78$ \\
\hline Raceł & & & & & & & & & & & & & & & & \\
\hline White & 4613 & 928 & 1.00 & - & 4613 & 1775 & 1.00 & - & 4613 & 1524 & 1.00 & - & 4613 & 532 & 1.00 & \\
\hline Non white & 75 & 17 & 0.99 & $0.62-1.59$ & 75 & 34 & 1.09 & $0.77-1.54$ & 75 & 29 & 1.03 & $0.71-1.48$ & 75 & 11 & 1.25 & $0.72-2.19$ \\
\hline Marital status§ & & & & & & & & & & & & & & & & \\
\hline Married & 3740 & 726 & 1.00 & - & 3740 & 140 & 1.00 & - & 3740 & 1166 & 1.00 & - & 3740 & 408 & 1.00 & \\
\hline Other than married ${ }^{\dagger}$ & 935 & 218 & 0.92 & $0.79-1.07$ & 935 & 70 & 1.59 & $1.18-2.12$ & 935 & 383 & 1.05 & $0.94-1.19$ & 935 & 134 & 1.04 & $0.86-1.27$ \\
\hline Education** & & & & & & & & & & & & & & & & \\
\hline Associate + Bachelor's Degree & 1781 & 302 & 1.00 & & 1781 & 69 & 1.00 & - & 1781 & 525 & 1.00 & - & 1781 & 182 & 1.00 & \\
\hline Master & 2516 & 546 & 0.97 & $0.84-1.12$ & 2516 & 112 & 0.96 & $0.71-1.31$ & 2516 & 887 & 0.87 & $0.78-0.98$ & 2516 & 308 & 0.93 & $0.77-1.13$ \\
\hline Education specialist degree & 307 & 79 & 1.02 & $0.80-1.31$ & 307 & 27 & 1.55 & $0.98-2.46$ & 307 & 113 & 0.77 & $0.63-0.95$ & 307 & 45 & 1.00 & $0.72-1.39$ \\
\hline Doctorate degree & 68 & 17 & 1.05 & $0.64-1.73$ & 68 & 2 & 0.79 & $0.20-3.13$ & 68 & 23 & 0.77 & $0.51-1.18$ & 68 & 6 & 0.61 & $0.26-1.44$ \\
\hline Years worked as a teachert† & & & & & & & & & & & & & & & & \\
\hline $0-10$ years & 1371 & 318 & 1.00 & & 1371 & 85 & 1.00 & - & 1371 & 474 & 1.00 & - & 1371 & 183 & 1.00 & \\
\hline 10-19 years & 1414 & 297 & 0.88 & $0.73-1.05$ & 1414 & 54 & 0.58 & $0.39-0.87$ & 1414 & 494 & 0.92 & $0.79-1.06$ & 1414 & 151 & 0.80 & $0.62-1.03$ \\
\hline 20-29 years & 1103 & 200 & 0.82 & $0.65-1.04$ & 1103 & 45 & 0.74 & $0.45-1.19$ & 1103 & 355 & 0.90 & $0.75-1.08$ & 1103 & 123 & 0.92 & $0.67-1.26$ \\
\hline$>30$ years & 774 & 128 & 0.90 & $0.68-1.20$ & 774 & 26 & 0.96 & $0.51-1.80$ & 774 & 221 & 0.96 & $0.77-1.19$ & 774 & 83 & 1.14 & $0.79-1.65$ \\
\hline Years worked in current schoo & & & & & & & & & & & & & & & & \\
\hline $0-4$ years & 823 & 198 & 1.00 & & 823 & 43 & 1.00 & - & 823 & 298 & 1.00 & - & 823 & 111 & 1.00 & \\
\hline 5-9years & 575 & 115 & 0.90 & $0.71-1.14$ & 575 & 25 & 0.83 & $0.49-1.42$ & 575 & 199 & 1.00 & $0.83-1.21$ & 575 & 65 & 0.95 & $0.69-1.32$ \\
\hline 10-19 years & 719 & 158 & 1.04 & $0.79-1.35$ & 719 & 30 & 1.00 & $0.55-1.82$ & 719 & 244 & 0.97 & $0.79-1.20$ & 719 & 84 & 0.95 & $0.66-1.37$ \\
\hline$>20$ & 528 & 84 & 0.92 & $0.65-1.28$ & 528 & 18 & 0.91 & $0.43-1.92$ & 528 & 155 & 1.01 & $0.78-1.30$ & 528 & 51 & 0.92 & $0.59-1.44$ \\
\hline of events was & & & & & & & & & & & & & & & & \\
\hline married: living & adom & & & & 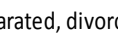 & wide & & & & & & & & & & \\
\hline$\ddagger$ Adjusted for non-response & & & & & & & & & & & & & & & & \\
\hline$\S$ Adjusted for gender, race an & & & & & & & & & & & & & & & & \\
\hline **Adjusted for gender, race, a & & & & & & & & & & & & & & & & \\
\hline t+Adjusted for gender, race, a & id education & & & & & & & & & & & & & & & \\
\hline F Adjusted for gender, race, a & ation, $j$ & & & & & & & & & & & & & & & \\
\hline
\end{tabular}


Table 2b. (continued) Rate Ratios for Non-Physical Violence Rates Per 100,000 Working Hours Per Year: Violence Against Educators Study

\begin{tabular}{|c|c|c|c|c|c|c|c|c|c|c|c|c|c|c|c|c|}
\hline \multirow[b]{3}{*}{ Variable } & \multicolumn{4}{|c|}{ Threat } & \multicolumn{4}{|c|}{ Sexual harassment } & \multicolumn{4}{|c|}{ Verbal abuse } & \multicolumn{4}{|c|}{ Bullying } \\
\hline & \multirow{2}{*}{$\begin{array}{r}\text { Number } \\
\text { Responses }\end{array}$} & \multicolumn{2}{|c|}{ Number Estimate } & \multirow{2}{*}{$95 \%$ C.I. } & \multirow{2}{*}{$\begin{array}{r}\text { Number } \\
\text { Responses }\end{array}$} & \multirow{2}{*}{$\begin{array}{l}\text { Number } \\
\text { Events* }\end{array}$} & \multirow{2}{*}{$\begin{array}{r}\text { Estimate } \\
\text { RR }\end{array}$} & \multirow[b]{2}{*}{ 95\% C.I. } & \multirow{2}{*}{$\begin{array}{r}\text { Number } \\
\text { Responses }\end{array}$} & \multirow{2}{*}{$\begin{array}{r}\text { Number } \\
\text { Events* }\end{array}$} & \multirow{2}{*}{$\begin{array}{r}\text { Estimate } \\
\text { RR }\end{array}$} & \multirow{2}{*}{$95 \%$ C.I } & \multirow{2}{*}{$\begin{array}{r}\text { Number } \\
\text { Responses }\end{array}$} & \multirow{2}{*}{$\begin{array}{r}\text { Number } \\
\text { Events* }\end{array}$} & \multirow{2}{*}{$\begin{array}{r}\text { Estimate } \\
\text { RR }\end{array}$} & \multirow[b]{2}{*}{ 95\% c.I. } \\
\hline & & Events* & RR & & & & & & & & & & & & & \\
\hline Type of school & & & & & & & & & & & & & & & & \\
\hline Public & 3935 & 775 & 1.00 & - & 3935 & 165 & 1.00 & - & 3935 & 1300 & 1.00 & - & 3935 & 446 & 1.00 & \\
\hline Public Alternative & 157 & 74 & 1.36 & $1.08-1.72$ & 157 & 25 & 1.93 & $1.25-2.99$ & 157 & 108 & 1.20 & $0 \quad 0.98-1.46$ & 157 & 36 & 1.31 & $0.94-1.82$ \\
\hline Public Charter & 85 & 25 & 1.13 & $0.75-1.70$ & 85 & 4 & 0.80 & $0.30-2.14$ & 85 & 34 & 1.01 & $10.71-1.43$ & 85 & 15 & 1.31 & $0.79-2.18$ \\
\hline Public Magnet & 63 & 24 & 1.33 & $0.88-2.02$ & 63 & 5 & 1.32 & $0.55-3.16$ & 63 & 29 & 1.03 & $\begin{array}{ll}3 & 0.71-1.50\end{array}$ & 63 & 7 & 0.66 & $0.31-1.44$ \\
\hline Private (Parochial) & 309 & 23 & 0.79 & $0.53-1.19$ & 309 & 4 & 0.58 & $0.21-1.59$ & 309 & 40 & 0.80 & 0 0.58-1.10 & 309 & 23 & 1.38 & $0.91-2.08$ \\
\hline Private(Non-Parochial) & 64 & 10 & 1.18 & $0.64-2.14$ & 64 & 1 & 0.36 & $0.03-4.13$ & 64 & 13 & 0.84 & $4 \quad 0.47-1.48$ & 64 & 7 & 1.30 & $0.60-2.80$ \\
\hline No one school type was most common & 29 & 11 & 1.20 & $0.63-2.25$ & 29 & 5 & 2.84 & $1.08-7.48$ & 29 & 16 & 1.04 & $4 \quad 0.62-1.74$ & 29 & 5 & 0.88 & $0.32-2.37$ \\
\hline Job classification§ & & & & & & & & & & & & & & & & \\
\hline Full-time & 1914 & 425 & 1.00 & - & 1914 & 92 & 1.00 & - & 1914 & 672 & 1.00 & - & 1914 & 240 & 1.00 & \\
\hline Part-time & 205 & 34 & 1.21 & $0.86-1.71$ & 205 & 9 & 1.34 & $0.66-2.71$ & 205 & 64 & 1.51 & 1 1.17-1.95 & 205 & 23 & 1.54 & $1.01-2.35$ \\
\hline Othert & 308 & 50 & 2.18 & $1.57-3.01$ & 308 & 7 & 1.64 & $0.74-3.63$ & 308 & 78 & 1.91 & 1 1.46-2.50 & 308 & 26 & 2.03 & $1.29-3.21$ \\
\hline Activity** & & & & & & & & & & & & & & & & \\
\hline Classroom teacher & 1837 & 332 & 1.00 & - & 1837 & 69 & 1.00 & - & 1837 & 563 & 1.00 & - & 1837 & 195 & 1.00 & \\
\hline $\begin{array}{l}\text { Administrator + Superintendent + Dean of } \\
\text { students }\end{array}$ & 71 & 23 & 1.01 & $0.63-1.63$ & 71 & 5 & 0.52 & $0.17-1.53$ & 71 & 33 & 0.87 & $\begin{array}{l}7 \\
0.59-1.28\end{array}$ & 71 & 14 & 1.18 & $0.64-2.16$ \\
\hline School psychologist & 18 & 7 & 1.25 & $0.56-2.80$ & 18 & 1 & 0.60 & $0.09-3.87$ & 18 & 9 & 1.12 & $20.57-2.21$ & 18 & 4 & 1.73 & $0.62-4.77$ \\
\hline School social worker & 33 & 7 & 1.31 & $0.63-2.72$ & 33 & 1 & 0.63 & $0.07-5.60$ & 33 & 11 & 1.17 & 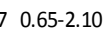 & 33 & 3 & 1.04 & $0.36-3.04$ \\
\hline Special education & 348 & 123 & 1.42 & $1.15-1.76$ & 348 & 20 & 0.95 & $0.57-1.58$ & 348 & 159 & 1.07 & $7 \quad 0.90-1.29$ & 348 & 60 & 1.16 & $0.86-1.56$ \\
\hline Department chair/ instructional leader & 11 & 2 & 1.65 & $0.42-6.45$ & 11 & 1 & 3.23 & $0.43-24.42$ & 11 & 2 & 0.99 & $90.26-3.79$ & 11 & 1 & 1.13 & $0.17-7.59$ \\
\hline $\begin{array}{l}\text { Teacher assistant/aide + advisor to extra- } \\
\text { curricular activities + other }\end{array}$ & 194 & 29 & 0.84 & $0.58-1.23$ & 194 & 9 & 1.20 & $0.58-2.48$ & 194 & 61 & 0.97 & 7 0.74-1.27 & 194 & 15 & 0.76 & $0.45-1.28$ \\
\hline No one activity was most common & 131 & 32 & 1.05 & $0.73-1.52$ & 131 & 10 & 1.20 & $0.59-2.44$ & 131 & 56 & 1.06 & $6 \quad 0.80-1.41$ & 131 & 19 & 1.01 & $0.62-1.65$ \\
\hline Class sizett & & & & & & & & & & & & & & & & \\
\hline$<10$ & 411 & 127 & 1.13 & $0.90-1.42$ & 408 & 29 & 1.60 & $0.97-2.66$ & 411 & 177 & 1.02 & $20.84-1.23$ & 411 & 75 & 1.19 & $0.88-1.60$ \\
\hline 10 to less than 25 & 952 & 179 & 1.00 & - & 950 & 32 & 1.00 & - & 952 & 281 & 1.00 & $0 \quad-$ & 952 & 109 & 1.00 & \\
\hline$>=25$ & 1069 & 205 & 0.80 & $0.65-1.00$ & 1068 & 47 & 1.28 & $0.79-2.07$ & 1069 & 360 & 0.92 & $2 \quad 0.78-1.09$ & 1069 & 107 & 0.79 & 0.59-1.05 \\
\hline Grade§§ & & & & & & & & & & & & & & & & \\
\hline Kindergartern-2nd grade & 371 & 48 & 1.00 & - & 371 & 7 & 1.00 & - & 371 & 75 & 1.00 & - & 371 & 33 & 1.00 & \\
\hline 3-6 grade & 530 & 97 & 1.04 & $0.74-1.47$ & 530 & 10 & 0.88 & $0.34-2.28$ & 530 & 137 & 1.01 & $10.76-1.34$ & 530 & 59 & 1.05 & $0.68-1.62$ \\
\hline 7-9 grade & 319 & 72 & 0.88 & $0.61-1.27$ & 319 & 22 & 2.00 & $0.85-4.71$ & 319 & 128 & 1.08 & $8 \quad 0.80-1.45$ & 319 & 50 & 1.13 & $0.72-1.78$ \\
\hline 10-12 grade & 219 & 58 & 0.98 & $0.66-1.44$ & 219 & 7 & 0.97 & $0.34-2.74$ & 219 & 93 & 1.05 & $5 \quad 0.77-1.45$ & 219 & 19 & 0.57 & $0.32-1.02$ \\
\hline No one grade level was most frequently taught & 982 & 234 & 0.99 & $0.73-1.36$ & 982 & 62 & 1.89 & $0.86-4.15$ & 982 & 380 & 1.07 & $\begin{array}{l}7 \\
0.83-1.38\end{array}$ & 982 & 128 & 0.88 & 0.59-1.32 \\
\hline * The number of events was established per edu & ucator & & & & & & & & & & & & & & & \\
\hline & & & & & & & & & & & & & & & & \\
\hline ¥ Adjusted for gender, race, age, merry status, $\mathrm{e}$ & ducation, job & 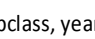 & teach & & & & & & & & & & & & & \\
\hline$\S$ Adjusted for gender, race, age, merry status, e & ducation, sch & hool type, & de and $y$ & year work & & & & & & & & & & & & \\
\hline ** Adjusted for gender, race, age, merry status, & education, jo & obclass, ye & a a teac & cher, year & worked and sc & school typ & & & & & & & & & & \\
\hline +† Adjusted for gender, race, age, education, job & bclass, year as & is a teacher & ear work & rked and sc & chool type anc & nd activity & & & & & & & & & & \\
\hline$\S \S$ Adjusted for gender, race, age, merry status, & education, jo & & & & & & & & & & & & & & & \\
\hline
\end{tabular}


Appendices 


\section{Appendix A}

Phase 1 (Comprehensive) Initial cover letter 


\section{PHASE 1 (COMPREHENSIVE) INITIAL COVER LETTER}

Date

Name

Address

Address

Dear $<<$ Teacher

Name $>$ :

Thank you for returning your form to us indicating you have worked as an educator in Minnesota in the past twelve months. At this time, we are following up regarding the Minnesota Educators' Study. We have enclosed an information sheet on the back of this letter, regarding the purpose and methods of this study, and about the drawing of 250 randomly selected individuals for the $\$ 100$ savings bonds. The opportunity for receiving a bond will be at least 1 in 75 individuals; however, this will vary based on the number of respondents. Individuals who are awarded the bonds will be notified at the completion of the data collection for both phases of the study (approximately May 2006).

For this initial portion of the study, participation includes completing and returning the enclosed questionnaire whether or not you experienced either physical or non-physical violence. Involvement in this study is voluntary, and choosing not to participate will not affect your future relations with any of the persons or institutions involved in this effort.

If you have any questions, please contact us at 612-626-4801 or toll free at 1-877-70-TEACH (1-877-708-3224). If you have any questions or concerns regarding this study and would like to talk to someone other than the researchers, you are encouraged to contact the Research Subjects' Advocate Line, D528 Mayo, 420 Delaware St. Southeast, Minneapolis, Minnesota 55455; (612) 625-1650. We look forward to your potential contribution to this important study and would appreciate your completing and returning the questionnaire by $<<$ date $>>>$. A postage-paid, return envelope is included for your convenience.

Sincerely,

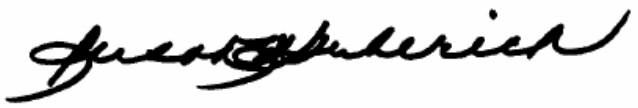

Susan Goodwin Gerberich, Ph.D.

Principal Investigator

Regional Injury Prevention Research Center and

Center for Violence Prevention and Control

Division of Environmental Health Sciences

University of Minnesota

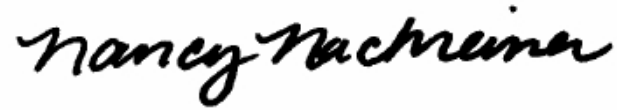

Nancy Nachreiner, Ph.D., R.N. Project Director 


\section{Appendix B}

Data Collection Materials: Minnesota Educators' Study: Phase 1 


\title{
Minnesota Educators' Study: Phase I
}

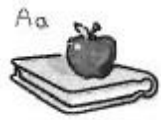 \\ Center for Violence Prevention and Control \\ Division of Environmental Health Sciences \\ School of Public Health, University of Minnesota \\ MMC 807, 420 Delaware St. SE \\ Minneapolis, MN 55455
}

Telephone: 612-626-4801; or toll free: 1-877-70-TEACH (1-877-708-3224)

Confidentiality - The information that you provide will be kept strictly confidential and no information that could personally identify you or the facility in which you work(ed) will ever be made public. Only investigators at the University of Minnesota will ever have access to this information. If there is any question you do not wish to answer, please mark an $\mathrm{X}$ on the question number, and continue to the next question. 
Drawing - We are providing savings bonds valued at $\$ 100$ each to 250 randomly selected individuals. You are not required to complete the questionnaire to be eligible for this drawing; however, you do need to check yes or no below, and return this questionnaire in the envelope provided.

1 Yes, include me in the savings bond drawing $2 \square$ No, do not include me in the savings bond drawing

1. Are you the person to whom this questionnaire was sent?

1 $\square$ Yes $2 \square$ No $\rightarrow$ Please call 1-877-708-3224 toll free, so that we may clarify the situation.

2. What is today's date?

$\overline{\text { month }} \frac{1}{\text { day }} \frac{}{\text { year }}$

3. Did you work as a licensed educator for a school (Kindergarten through Twelfth Grade), for any amount of time, in Minnesota, in the 12 months prior to tuday's date?

1 $\square$ Yes $2 \square$ No $\longrightarrow$ IF NO, Thank you for taking the time to respond. Please stop here and return the questionnaire in the enclosed envelope.

IF YES, Please continue to the next page. 
4. We would like to know about the hours you worked, as a licensed educator in Minnesota, for the 12 months prior to today's date. Below is a calendar to prompt your memory about your past work schedule. Please write in the number of days worked each month, and average number of hours you worked per day. If you did not work as a licensed educator during a specific month, please write "0." Please include the time you spent in direct student contact, preparation time, and other school-related assignments.

EXAMPLE: May 2004 is completed below as an example. This example shows the educator worked 18 days during May 2004, and worked on average 9 hours per day. Please complete the days and hours you worked for each of the previous 12 months.

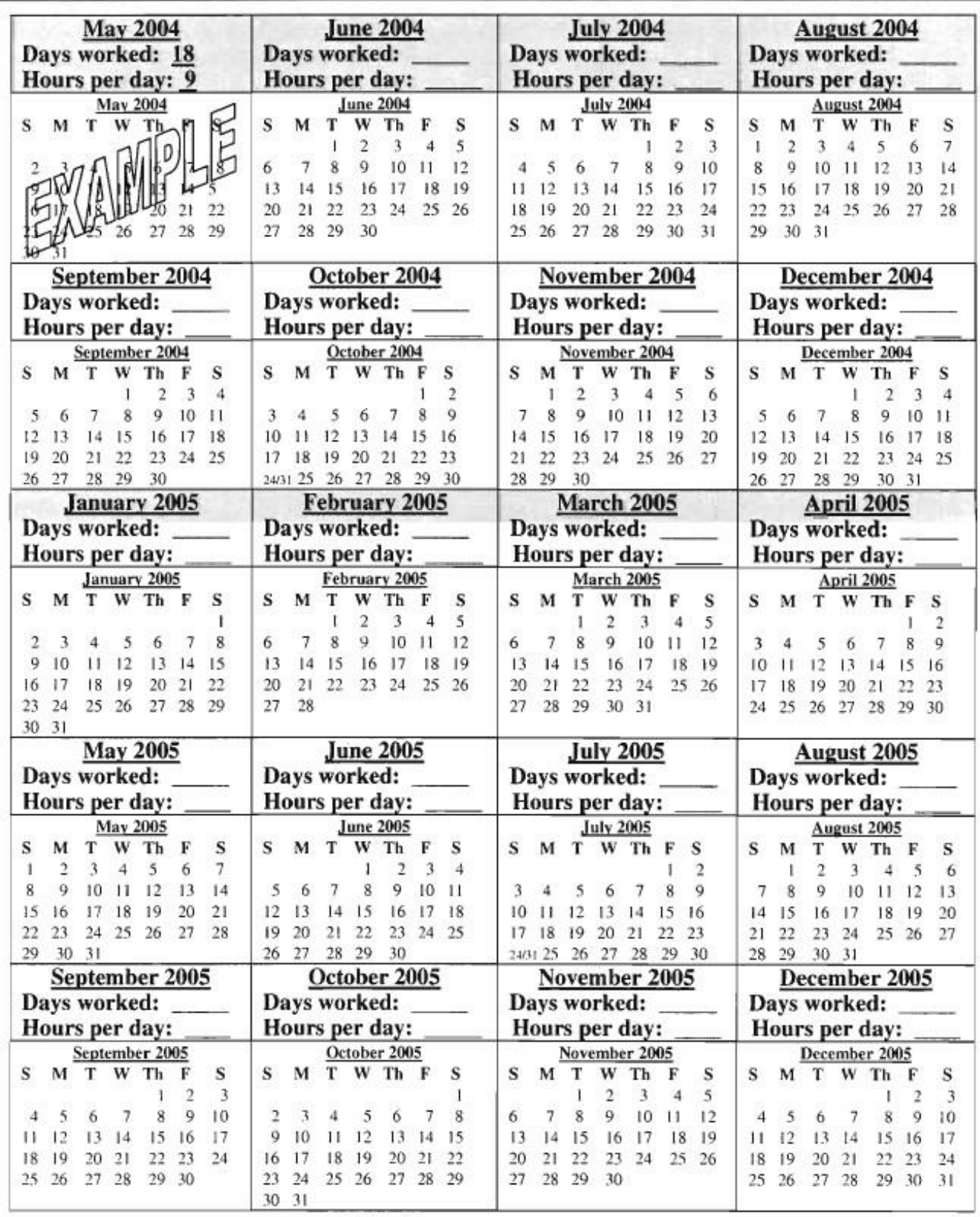


5. In what type(s) of school(s) did you work in the 12 months prior to today's date? Check all that apply.

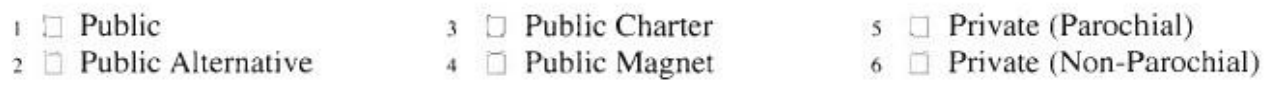

6. In what type(s) of school did you work the most time in the 12 months prior to today's date? Check one.

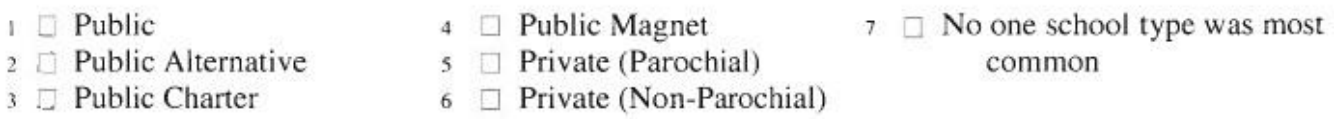

7. Consider the school where you worked the most time in the past 12 months. What is the total length of time that you have worked in that school? Please indicate the number of years and/or months. year(s) month(s)

8. What grade level(s) were taught in the school where you worked the most time? Check all that apply.

$\begin{array}{llll}1 \square \text { Kindergarten } & 6 \square \text { Fifth Grade } & 11 \square \text { Tenth Grade } \\ 2 \square \text { First Grade } & 7 \square \text { Sixth Grade } & 12 \square \text { Eleventh Grade } \\ 3 \square \text { Second Grade } & 8 \square \text { Seventh Grade } & 13 \square \text { Twelfth Grade } \\ 4 \square \text { Third Grade } & 9 \square \text { Eighth Grade } & & \\ 5 \square \text { Fourth Grade } & 10 \square \text { Ninth Grade } & \end{array}$

9. What was your job classification in the school where you worked the most time? Check one.

1 Full-time contract

$2 \square$ Part-time contract

3 Long-call substitute
$4 \square$ Building substitute (assigned daily to one building to cover classes as needed)

$s \square$ All other substitutes (day-to-day, short call, etc.)

10. Please check all of your professional activities in the 12 months prior to today's date. Check all that apply.

Classroom teacher
Librarian/Media Specialist
School Nurse
Administrator
Superintendent
Dean of Students
Teacher Assistant/Aide
Advisor to Extra-Curricular Activities

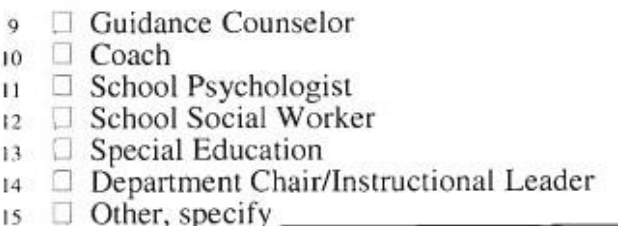

11. Please check your one most frequent activity in the $\mathbf{1 2}$ months prior to today's date. Check one.

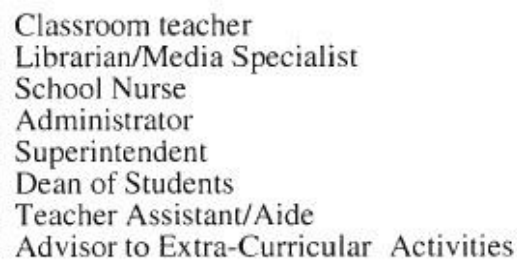

Guidance Counselor

Coach

School Psychologist

School Social Worker

Special Education

Department Chair/Instructional Leader

Other, specify

No one activity was most common. 
12. Did you teach students in a classroom or other setting in which instruction occurred in the $\underline{12}$ months prior to today's date? Check one.

$1 \square$ Yes $\quad 2 \square$ No $\longrightarrow$ IF NO, go to Question 19.

13. What grade level(s) did you teach in the 12 months prior to today's date? Check all that apply.

$\begin{array}{ll}1 & \text { Kindergarten } \\ 2 & \text { First Grade } \\ 3 & \text { Second Grade } \\ 4 & \text { Third Grade } \\ 5 & \text { Fourth Grade }\end{array}$

\begin{tabular}{|c|c|}
\hline 6 & Fifth Grade \\
\hline 7 & Sixth Grade \\
\hline 8 & Seventh Grade \\
\hline & $\begin{array}{l}\text { Eighth Grade } \\
\text { Ninth Grade }\end{array}$ \\
\hline
\end{tabular}

$11 \square$ Tenth Grade

$12 \square$ Eleventh Grade

$13 \square$ Twelfth Grade

14. What grade level did you teach most frequently in the $\mathbf{1 2}$ months prior to today's date? Check one.

\begin{tabular}{|c|c|c|c|c|}
\hline I Kindergarten & 6 & $\square$ Fifth Grade & & Tenth Grade \\
\hline First Grade & 7 & Sixth Grade & 12 & Eleventh Grade \\
\hline Second Grade & 8 & Seventh Grade & $13 \square$ & Twelfth Grade \\
\hline $\begin{array}{l}4 \\
5 \\
5 \text { Fourth Grade }\end{array}$ & $\begin{array}{l}9 \\
10\end{array}$ & $\begin{array}{l}\text { Eighth Grade } \\
\text { Ninth Grade }\end{array}$ & $14 \square$ & $\begin{array}{l}\text { No one grade level was most } \\
\text { frequently taught. }\end{array}$ \\
\hline
\end{tabular}

15. What topic(s) did you teach in the 12 months prior to today's date? Check all that apply.
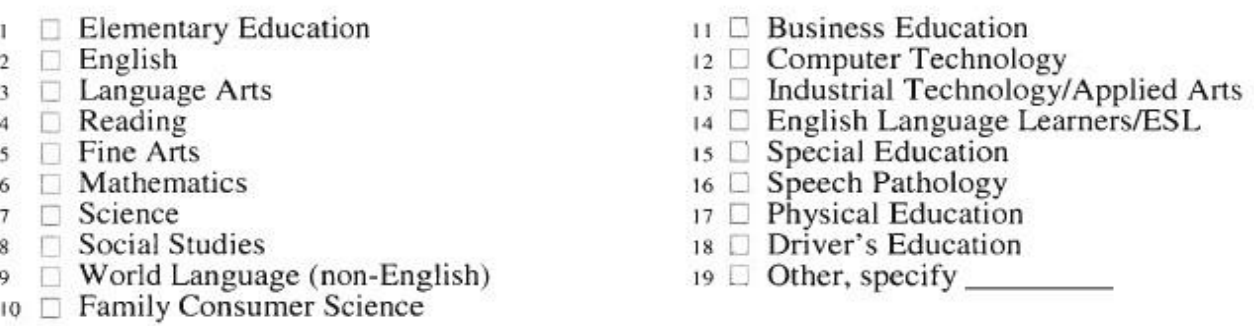

16. What topic did you teach most frequently in the 12 months prior to today's date? Check one.

Elementary Education
English
Language Arts
$\square$ Reading
Fine Arts
Mathematics
Science
$\square$ Social Studies
World Language (non-English)
Family Consumer Science

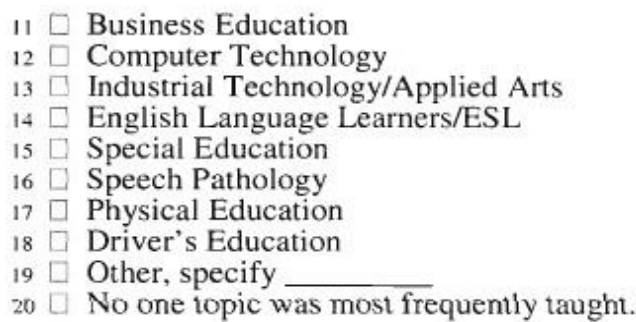

17. What was your typical class size in the 12 months prior to today's date? Check one.

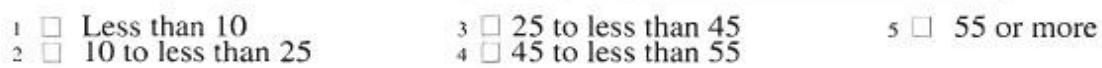

18. Were there typically any other personnel (licensed educators or non-educators), besides yourself, in your classroom (or other setting in which instruction occurred) in the 12 months prior to today's date? Check only one. $1 \square$ Yes $2 \square$ No

The next section pertains to work-related violence events. Work-related includes any activities associated with jobs or events that occur in your work environment; work-related travel is included. Work-related violence is defined as the intentional use of physical force or emotional abuse, against an employee, that results in physical or emotional injury and consequences. This includes physical assault threat, sexual harassment, verbal abuse, and bullying. 


\section{PHYSICAL VIOLENCE}

Physical assault occurs when you are hit, slapped, kicked, pushed, choked, grabbed, sexually assaulted, or otherwise subjected to physical contact intended to injure or harm you. Questions 19 through 46 relate to physical assault only; questions about threat, sexual harassment, verbal abuse, and bullying will follow.

19. Were you the target of a work-related physical assault at any time during the 12 months prior to today's date? Check YES or NO.

$$
1 \square_{1} \text { Yes } \quad 2 \square \mathrm{No} \longrightarrow \underline{\text { IF NO, go to Page II. }}
$$

20. Please describe the(se) event(s): Check one.

1 Single event (one-time occurrence)

2 - Multiple events (specific separate events that you can describe)

3 Ongoing events (events that occur with such frequency that you are only able to describe them in general) $\longrightarrow$ If ongoing, please estimate the number of events in the past 12 months: _ number of violent events

If you experienced single or multiple events, please describe each event (1-4) below; if you experienced ongoing violence, please complete the Event 1 columns regarding how the events occurred, in general, and please write in the earliest date the events occurred in the past 12 months.

Please provide the following information for each physical assault event that happened to you during the 12 months prior to today's date.

- If you experienced more than one event, it may be easier to first complete Questions 21 through 46 for Event 1 , and then go back and complete Questions 21 through 46 for Event 2, etc.

- If you experienced more than 4 events in the previous 12 months, please provide information for Questions 21 through 46 for each event on a separate sheet of paper, or call 1-877-708-3224 toll free, or 612-626-480I, for additional copies of this questionnaire.

21. Date(s) of physical assault(s): Fill in month and year. If unsure of exact month, please give your best estimate.

22. Time(s) of physical assault(s): Check all that apply for each event. 1 During regular school hours 2 Before or after regular school hours 8 Unsure

23. What was(were) the location(s) of the physical assault(s)? Check all that apply for each event. iClassroom 2 Hallway How many people were in the room?

${ }_{3}$ Parking area

4 Stairway

5 Staff or student lounge

6 Away from school property

7 In a vehicle (bus, etc.)

8 Athletic field/court

9 Extra curricular setting (stage, etc.)

10 Other

$$
\text { Specify }
$$

11 Unsure

24. Did only one person assault you? Check one for each event.

1 Yes

2 No (more than one)

\begin{tabular}{|c|c|c|c|}
\hline Event 1 & Event 2 & Event 3 & Event 4 \\
\hline-1 & -1 & 1 & -1 \\
\hline$\overline{\mathrm{mo}} . / \overline{\mathrm{yr}}$ & $\overline{\mathrm{mo}} \cdot / \mathrm{yr}$. & mo./yr. & $\overline{\mathrm{mo}} . / \mathrm{yr}$. \\
\hline $1 \square$ & 1口 & $1 \square$ & 10 \\
\hline 27 & $2 \square$ & $2 \square$ & $2 \square$ \\
\hline 8] & $8 \square$ & $8[1$ & 8[ \\
\hline $1 \square$ & 10 & 1] & 10 \\
\hline $2 \square$ & $2 \square$ & $2 \square$ & 2] \\
\hline $3 \square$ & $3 \square$ & $3 \square$ & $3 \square$ \\
\hline $4 \square$ & $4 \square$ & $4 \square$ & $4 \square$ \\
\hline $5 \square$ & $5 \square$ & $5 \square$ & $5 \square$ \\
\hline $6 \square$ & $6 \square$ & $6 \square$ & $6 \square$ \\
\hline $7 \square$ & $7 \square$ & $7 \square$ & $7 \square$ \\
\hline $8 \square$ & $8 \square$ & 8 & $8]$ \\
\hline $9 \square$ & $9 \square$ & $9 \square$ & $9 \square$ \\
\hline $10 \square$ & 107 & $10 \square$ & $10 \square$ \\
\hline $11 \square$ & $11 \square$ & $11 \square$ & $\mathrm{Ii} \square$ \\
\hline $1 \square$ & $1 \square$ & $1 \square$ & 10 \\
\hline $2 \square$ & $2 \square$ & 2 & 2 \\
\hline $8 \square$ & 8[] & $8 \square$ & $8 \square$ \\
\hline
\end{tabular}

8 Unsure 
25. What was(were) your relationship(s) with the person(s) who physically assaulted you? Check all that apply for each event.

1 Your current student

2 Your former student

3 Another currently enrolled student

4 Another formerly enrolled student

5 Someone you supervise

6 Another employee

7 Parent

8 Visitor

9 Trespasser

10 No professional relationship

"Other Specify

88 Unsure

26. What was(were) the gender(s) of the person(s) who physically assaulted you? Check all that apply for each event.

1 Male

2 Female

8 Unsure

27. Was(were) the perpetrator(s) of the physical assault of the same race/ethnicity as you? Check one for each event.

1 Yes

2 No

8 Unsure

28. In what age group(s) was(were) the person(s) who physically assaulted you? Check all that apply for each event.

1 Age less than 13 years

213 years to less than 16 years

316 years to less than 18 years

4 Adult

8 Unsure

29. Was(were) the person(s) who physically assaulted you impaired?

Check all that apply for each event.

1 Yes, because of injury or illness (physical impairment, hearing impairment, etc.)

2 Yes, because of disability (learning, emotional, autism, etc.) or developmental delay

3 Yes, under influence of alcohol, aerosols, or drugs (prescribed or non-prescribed)

4 Not impaired

5 Unsure

30. What did the person(s) use to physically assault you? Check all that

apply for each event.

1 Hands/Arms

2 Feet/Legs

3 Teeth

4 Genitals

5 Gun

6 Knife

7 Bodily Fluids (e.g., spit)

8 Other,

9 Unsure Specify

\begin{tabular}{|c|c|c|c|}
\hline Event 1 & Event 2 & Event 3 & Event 4 \\
\hline 18 & 10 & 10 & 10 \\
\hline $2 \square$ & $2 \Gamma$ & $2 \square$ & $2 \square$ \\
\hline $3 \square$ & $3 \square$ & $3 \square$ & $3 \square$ \\
\hline $4 \square$ & $4 \square$ & $4 \square$ & $4 \square$ \\
\hline $5 \square$ & $5 \square$ & $5 \square$ & $5 \square$ \\
\hline $6 \square$ & $6 \square$ & $6 \square$ & $6 \square$ \\
\hline $7 \square$ & $7 \square$ & $7 \square$ & 7 C \\
\hline $8 \square$ & $8 \square$ & $8 \square$ & $8 \square$ \\
\hline $9[\square$ & $9 \square$ & $9 \square$ & $9 \square$ \\
\hline $10 \square$ & $10[\bar{C}$ & $10 \square$ & $10 \square$ \\
\hline 110 & $19 \square$ & $11 \square$ & 110 \\
\hline $88 \square$ & $88 \square$ & $88 \square$ & $88 \square$ \\
\hline $1[\square$ & $1 \square$ & 1] & $1 \square$ \\
\hline $2 \square$ & $2 \square$ & $2 \square$ & $2 \square$ \\
\hline $8 \square$ & $8 \square$ & $8 \square$ & $8 \square$ \\
\hline 1$]$ & 10 & $1 \square$ & 1口 \\
\hline $2 \square$ & $2 \square$ & $2 \square$ & $2 \square$ \\
\hline $8 \square$ & $8 \square$ & $8 \square$ & $8 \square$ \\
\hline 10 & 1] & $1 \square$ & $1 \square$ \\
\hline $2 \square$ & $2 \square$ & $2 \square$ & $2 \square$ \\
\hline $3 \square$ & 3】 & $3 \square$ & $3 \square$ \\
\hline $4 \square$ & $4 \square$ & $4 \square$ & $4 \square$ \\
\hline $8 \square$ & $8 \square$ & $8 \square$ & $8 \square$ \\
\hline 10 & 10 & 1 & $1 \square$ \\
\hline $2 \square$ & $2 \square$ & 2\llcorner & $2 \square$ \\
\hline $3 \square$ & $3 \square$ & $3 \square$ & 3[ \\
\hline $4 \square$ & $4 \square$ & $4 \square$ & $4 \square$ \\
\hline $5 \square$ & 5[ & $5 \square$ & $5 \square$ \\
\hline 1$]$ & 10 & 10 & $1 \mathrm{I}$ \\
\hline $2 \square$ & $2 \square$ & $2 \square$ & $2 \square$ \\
\hline 3[ & $3 \square$ & $3 \square$ & $3 \square$ \\
\hline $4 \square$ & $4 \square$ & $4 \square$ & $4 \square$ \\
\hline $5 \square$ & 5 & 5口 & $5 \square$ \\
\hline $6 \square$ & $6 \square$ & $6 \square$ & $6 \square$ \\
\hline $7 \square$ & $7 \square$ & 7[] & $3 \square$ \\
\hline $8 \square$ & $8 \square$ & $8 \square$ & $8 \square$ \\
\hline $9 \square$ & $9 \square$ & 9[ & 90 \\
\hline
\end{tabular}


31. Was(were) the event(s) witnessed? Check one for each event. 1 Yes

2 No

8 Unsure

32. What was(were) the type(s) of physical injury? Check all that apply for each event.

I Abrasion

2 Amputation

3 Asphyxia

4 Bite

5 Bruise/contusion

6 Burn

7 Concussion (Loss of consciousness/awareness)

8 Crushing/mangling

9 Cut/laceration/scratch

10 Fracture/dislocation

II Nerve injury

12 Penetration injury including puncture

13 Poisoning

14 Rupture

is Sexual assault

$16 \mathrm{Sprain} / \mathrm{strain}$

17 Temporary discoloration/slap mark

18 Torn ligament

19 Other

20 None

Specify

33. What body part(s) was(were) injured? Check all that apply for each event.

1 Head/skull/brain

2 Face (forehead, cheek, nose, lip, jaw, ear)

3 Eye/eyelid

4 Teeth

5 Neck (cervical area)

6 Back (muscles, skin)

7 Internal chest

8 External chest (muscles, skin)

${ }_{9}$ Spinal cord/spine (vertebrae, sacrum, tailbone, coccyx, disks)

10 Internal abdomen

11 External abdomen (muscles, skin)

12 Shoulder/collar bone, shoulder blade

13 Arm/elbow/wrist

$14 \mathrm{Hand} /$ fingers/thumb(s)

15 Internal hips/pelvis (uterus, ovaries, bladder, rectum)

16 External hips/pelvis (muscles, skin)

17 Buttocks

18 Genitalia

19 Leg (thigh, shin, calf, knee, ankle)

20 Foot/heel, toes

${ }_{21}$ General systems (e.g., respiratory, cardiovascular)

22 Other

23 None

$$
\text { Specify }
$$

\begin{tabular}{|c|c|c|c|}
\hline Event 1 & Event 2 & Event 3 & Event 4 \\
\hline 10 & $1]$ & 다 & $1 \square$ \\
\hline $2 \square$ & $2 \square$ & $2 \square$ & $2 \square$ \\
\hline $8 \square$ & 8 & $8 \square$ & $8 \square$ \\
\hline $1 \square$ & $1]$ & 1ㅁ & $1 \square$ \\
\hline $2 \square$ & 2】 & $2 \square$ & $2 \square$ \\
\hline $3 \square$ & $3 \square$ & $3 \square$ & $3 \square$ \\
\hline $4 \square$ & $4 \square$ & $4 \square$ & $4 \square$ \\
\hline $5 \square$ & $5 \square$ & $5 \square$ & $5 \square$ \\
\hline $6 \square$ & $6 \square$ & $6 \square$ & $6 \square$ \\
\hline $7 \square$ & $7 \square$ & $7 \square$ & $7 \square$ \\
\hline $8 \square$ & $8 \square$ & $8 \square$ & $8 \square$ \\
\hline $9 \square$ & 9] & $9 \square$ & $9 \square$ \\
\hline $10 \square$ & $10 \square$ & $10 \square$ & $10 \square$ \\
\hline $11 \square$ & $11 \square$ & $11 \square$ & $11 \square$ \\
\hline $12 \square$ & $12 \square$ & $12 \square$ & $12 \square$ \\
\hline $13 \square$ & ${ }_{13} \square$ & $13 \square$ & $13 \square$ \\
\hline $14 \square$ & $14 \square$ & $14 \square$ & $14 \square$ \\
\hline $15 \square$ & $15 \square$ & $15 \square$ & $15 \square$ \\
\hline 160 & $16 \square$ & $16 \square$ & $16 \square$ \\
\hline 17口 & $17 \square$ & $17 \square$ & $17 \square$ \\
\hline $18 \square$ & $18 \square$ & $18 \square$ & $18 \square$ \\
\hline 19[] & $19 \square$ & $19 \square$ & $19 \square$ \\
\hline $20 \square$ & $20 \square$ & $20 \square$ & $20 \square$ \\
\hline $1 \square$ & $1 \square$ & $1 \square$ & $1 \square$ \\
\hline $2 \square$ & $2 \square$ & $2 \mathrm{C}$ & $2 \square$ \\
\hline $3 \square$ & $3[$. & $3 \square$ & $3 \square$ \\
\hline $4 \square$ & $4 \square$ & $4 \square$ & $4 \square$ \\
\hline $5 \square$ & $5 \square$ & $5 \square$ & $5 \square$ \\
\hline $6 \square$ & $6 \square$ & $6 \square$ & $6 \square$ \\
\hline $7 \square$ & $7 \square$ & $7 \square$ & $7 \square$ \\
\hline $8 \square$ & $8 \square$ & $8 \square$ & $8 \square$ \\
\hline $9 \square$ & $9 \square$ & $9 \square$ & $9 \square$ \\
\hline $10 \square$ & $10 \square$ & $10 \square$ & $10 \square$ \\
\hline $11 \square$ & $11 \square$ & $11 \square$ & $11 \square$ \\
\hline $12 \square$ & $12 \square$ & $12 \square$ & $12 \square$ \\
\hline 13[ & $13 \square$ & $13 \square$ & 13] \\
\hline $14 \square$ & $14 \square$ & $14 \square$ & $14 \square$ \\
\hline 15口 & $15 \square$ & $15 \square$ & $15 \square$ \\
\hline $16 \square$ & $16 \square$ & $16 \square$ & $16 \square$ \\
\hline $17 \square$ & $17 \square$ & $17 \square$ & $17 \square$ \\
\hline $18 \square$ & $18 \square$ & $18 \square$ & $18 \square$ \\
\hline $19 \square$ & $19 \square$ & $19 \square$ & $19 \square$ \\
\hline $20 \square$ & $20 \square$ & $20 \square$ & $20 \square$ \\
\hline $21 \square$ & $21 \square$ & $21 \square$ & $21 \square$ \\
\hline $22 \square$ & $22 \square$ & $22 \square$ & $22 \square$ \\
\hline $23 \square$ & $23 \square$ & $23 \square$ & $23 \square$ \\
\hline
\end{tabular}


34. As a result of the physical assault(s), what types of symptoms/ feelings have you experienced? Check all that apply for each event. 1 Anger

2 Depression

3 Sadness

4 Fatigue

${ }_{5}$ Frustration

6 Fear/anxiety/stress

7 Shame/Low self-esteem/Low self-confidence

8 Stress-related physical symptoms (headaches, stomach problems, etc.)

9 Difficulty sleeping

10 Nightmares

11 Hallucinations

12 Flashbacks

13 Difficulty concentrating

14 Irritability

15 Intrusive thoughts about the event

16 Hyperarousal/Hypervigilance/Overly cautious

17 Avoidance of any reminders of the event

18 Resurfacing memories of previous trauma

19 Withdrawal from contact with people

20 Other,

21 None

$$
\text { Specify }
$$

35. Did any of your symptoms (identified in Question 34) last for at least 1 month? Check one for each event.

IYes

2 No

31 month has not passed since the event occurred

36. Did any of your symptoms (identified in Question 34) last for 6 months or more? Check one for each event.

1 Yes

2 No

36 months have not passed since the event occurred

37. Are you currently experiencing any persistent problems or svmptoms related to this(these) event(s)? Check one for each event. 1 Yes 2 No

If YES, please describe

38. Were you treated by any of the following as a result of this(these) event(s)? Check all that apply for each event.

i No treatment

2 Physician (non-Psychiatrist)

3 Dentist

4 Chiropractor

5 Nurse/Nurse Practitioner/Nurse Clinician/Physician's Assistant

6 Psychiatrist/Psychologist/Therapist

7 Physical/Occupational Therapist

8 Athletic Trainer

9 Paramedics/Emergency Medical Technician

10 Homeopathic, Alternative, or Non-traditional medicine provider

11 Treated yourself

12 Other,

$$
\text { Specify }
$$

\begin{tabular}{|c|c|c|c|}
\hline Event 1 & Event 2 & Event 3 & Event 4 \\
\hline $1 \square$ & 10 & 1] & 1口 \\
\hline $2 \square$ & $2 \square$ & $2 \square$ & $2 \square$ \\
\hline $3 \square$ & $3 \square$ & $3 \square$ & $3 \square$ \\
\hline $4 \square$ & $4 \square$ & $4 \square$ & $4 \square$ \\
\hline 50 & $5 \square$ & 5口 & $5 \biguplus$ \\
\hline 6.] & $6 \square$ & $6]$ & 6] \\
\hline 70 & $7 \square$ & $7 \square$ & $7 \square$ \\
\hline $8 \square$ & $8 \square$ & $8 \square$ & $81]$ \\
\hline $9 \square$ & $9[\square$ & $9 \square$ & 9ㅁ \\
\hline $10 \square$ & $10 \square$ & $10 \square$ & $10 \square$ \\
\hline 110 & $11 \square$ & 110 & $11 \square$ \\
\hline $12 \square$ & $12 \square$ & $12 \square$ & $12 \square$ \\
\hline 13 & $13 \square$ & $13 \square$ & $13 \square$ \\
\hline $14 \square$ & $14 \square$ & $14 \square$ & $14 \square$ \\
\hline $15 \square$ & $15 \square$ & $15 \square$ & 15[] \\
\hline $16 \square$ & $16 \square$ & $10 \square$ & $16 \square$ \\
\hline $17 \square$ & $17 \square$ & 17느 & 17口 \\
\hline $18 \square$ & $18 \square$ & $18 \square$ & $18 \square$ \\
\hline 19】 & 19[] & $19 \square$ & 19】 \\
\hline $20 \square$ & $20 \square$ & $20 \square$ & $20 \square$ \\
\hline $21 \square$ & $21 \square$ & 210 & $21 \square$ \\
\hline 10 & 10 & 1口 & 10 \\
\hline $2 \square$ & $2 \square$ & $2 \square$ & $2 \square$ \\
\hline $3 \square$ & $3 \square$ & $3 \square$ & $3 \mathrm{D}$ \\
\hline 10 & 10 & 10 & 10 \\
\hline $2 \square$ & $2 \square$ & $2 \square$ & $2 \square$ \\
\hline $3 \square$ & $3 \square$ & $3 \square$ & $3 \mathrm{D}$ \\
\hline $1 \square$ & $1 D$ & $1 \square$ & 10 \\
\hline $2 \square$ & $2 \square$ & $2 \square$ & $2 \square$ \\
\hline $1 \square$ & $1 \square$ & 1D & $1 \square$ \\
\hline $2 \square$ & $2 \square$ & $2 \square$ & $2 \square$ \\
\hline $3 \square$ & 3[] & $3 \square$ & $3 \square$ \\
\hline $4 \square$ & $4 \square$ & $4 \square$ & $4 \square$ \\
\hline $5 \square$ & $5 \square$ & $5 \square$ & $5 \square$ \\
\hline $6 !$ & $6 \square$ & $6 \square$ & $6 \square$ \\
\hline $7 \square$ & $7 \square$ & $7 \square$ & $7 \square$ \\
\hline $8 \square$ & $8 \square$ & $8 \square$ & 8[] \\
\hline $9 \square$ & $9 \square$ & $9 \square$ & $9 \square$ \\
\hline $10 \square$ & $10 \square$ & $10 \square$ & $10 \square$ \\
\hline $11 \square$ & 11口 & $11 \square$ & $11 \square$ \\
\hline $12 \square$ & $12 \square$ & $12 \square$ & $12 \square$ \\
\hline
\end{tabular}


39. Were you admitted to a hospital as a result of this(these) event(s)? Check one for each event.

1 Yes 2 No

If YES, for how many days?

40. What changes in vour work situation occurred as a result of this(these) event(s)? Check all that apply for each event.

I Quit your job

2 Voluntary transfer to another location

3 Involuntary transfer to another location

4 Leave of absence

5 Restriction/modification of work activities

6 No changes

7 Other, Specify

41. How long were regular work and/or non-work activities restricted as a result of this(these) event(s)? Check one for each event.

1 Less than 4 hours

24 hours to less than 1 day

31 day to less than 3 days

43 days to less than 7 days

57 days to less than 14 days

614 days to less than 1 month

71 month to less than 3 months

83 months or more

9 No restriction

42. Are your regular activities still restricted as a result of this(these) event(s)? Check one for each event.

1 Yes

$2 \mathrm{No}$

43. As a result of this(these) event(s), how long were you absent from work? Check one for each event.

I Less than 4 hours

24 hours to less than 1 day

31 day to less than 3 days

43 days to less than 7 days

57 days to less than 14 days

614 days to less than 1 month

71 month to less than 3 months

83 months or more

9 No absence

44. How would you rate the severity of your symptom(s)/problem(s) resulting from this(these) event(s)? Consider any limitation of your abilities (physical/mental) and/or activities. Check one for each event.

1 No limitations of abilities/activities

2 Some limitations of abilities/activities

3 Moderate limitations of abilities/activities

4 Severe limitations of abilities/activities

5 Disabling -inability to function

\begin{tabular}{|c|c|c|c|}
\hline Event 1 & Event 2 & Event 3 & Event 4 \\
\hline $1 \square$ & $1 \square$ & $1 \square$ & $1 \square$ \\
\hline $2 \square$ & $2 \square$ & $2 \square$ & $2 \square$ \\
\hline $1 \square$ & $1 \square$ & 10 & $1 \square$ \\
\hline $2 \square$ & $2 \square$ & $2 \square$ & $2 \square$ \\
\hline 3曰 & $3 \square$ & $3 \square$ & $3 \square$ \\
\hline $4 \square$ & $4 \square$ & $4 \square$ & $4 \square$ \\
\hline $5 \square$ & $5 \square$ & $5 \square$ & $5 \square$ \\
\hline $6 \square$ & $6 \square$ & $6 \square$ & $6 \square$ \\
\hline $7 \square$ & $7 \square$ & $7 \square$ & 70 \\
\hline $1[\mathrm{C}$ & $1 \square$ & $1 \square$ & $1 \square$ \\
\hline $2 \square$ & 2[] & $2 \square$ & $2 \square$ \\
\hline $3 \square$ & $3 \square$ & $3 \square$ & $3 \square$ \\
\hline $4 \square$ & $4 \square$ & $4 \square$ & $4 \square$ \\
\hline $5 \square$ & $5 \square$ & $5 \square$ & $5 \square$ \\
\hline $6 \square$ & $6 \square$ & $6 \square$ & $6 \square$ \\
\hline $7 \square$ & $7 \square$ & $7 \square$ & 70 \\
\hline $8 \square$ & $8 \square$ & $8 \square$ & $8 \square$ \\
\hline $9 \square$ & $9 \square$ & $9 \square$ & $9 \square$ \\
\hline 10 & $1 \square$ & $1 \square$ & 10 \\
\hline $2 \square$ & $2 \square$ & 2[] & $2 \square$ \\
\hline $1 \square$ & $1 \square$ & $1 \square$ & $1 \square$ \\
\hline $2 \square$ & $2 \square$ & $2 \square$ & $2 \square$ \\
\hline $3 \square$ & $3 \square$ & $3 \square$ & $3 \square$ \\
\hline $4 \square$ & $4 \square$ & $4 \square$ & $4 \square$ \\
\hline$s \square$ & $5 \square$ & $5 \square$ & $5 \square$ \\
\hline $6 \square$ & $6 \square$ & 6]$. & $6 \square$ \\
\hline $7 \square$ & $7 \square$ & $7 \square$ & $7 \square$ \\
\hline $8 \square$ & 80 & 81 & $8 \square$ \\
\hline $9 \square$ & 90 & $9 \square$ & $9 \square$ \\
\hline $1 \square$ & $1 \square$ & $1 \square$ & । \\
\hline $2 \square$ & 20 & $2 \square$ & $2 \square$ \\
\hline $3 \square$ & $3 \square$ & $3 \square$ & $3 \square$ \\
\hline $4 \square$ & $4 \square$ & $4 \square$ & $4 \square$ \\
\hline$s \square$ & $5 \square$ & $5 \square$ & $s \square$ \\
\hline
\end{tabular}


45. Did you report the event(s) to administration? Check all that apply for each event.

1 Yes, orally

2 Yes, written

3 No, I did not report the event

If YES, in your opinion, was administration adequately responsive to your report?

4 Yes

5 No

6 Unsure

If $\underline{\mathrm{NO}}$, please check reason(s) that event(s) was(were) not reported.

7 Reporting takes too much time

8 Unaware of reporting process

9 Did not expect report to change situation

10 Did not feel necessary to report

11 Other

Specify

\begin{tabular}{|c|c|c|c|}
\hline Event 1 & Event 2 & Event 3 & Event 4 \\
\hline & & & \\
$1 \square$ & $1 \square$ & $1 \square$ & $1 \square$ \\
$2 \square$ & $2 \square$ & $2 \square$ & $2 \square$ \\
$3 \square$ & $3 \square$ & $3 \square$ & $3 \square$ \\
& & & \\
$4 \square$ & $4 \square$ & $4 \square$ & $4 \square$ \\
$5 \square$ & $5 \square$ & $5 \square$ & $5 \square$ \\
$6 \square$ & $6 \square$ & $6 \square$ & $6 \square$ \\
$7 \square$ & $7 \square$ & $7 \square$ & $7 \square$ \\
$8 \square$ & $8 \square$ & $8 \square$ & $8 \square$ \\
$9 \square$ & $9 \square$ & $9 \square$ & $9 \square$ \\
$10 \square$ & $10 \square$ & $10 \square$ & $10 \square$ \\
$11 \square$ & $11 \square$ & $11 \square$ & $11 \square$ \\
\hline
\end{tabular}

Comments regarding reporting:

46. Please describe how each event occurred. What were you doing just prior to the event? What was the person(s) who assaulted you doing just prior to the event? What triggered the event? Describe how you were assaulted. Please use additional paper as necessary.

Event 1:

Event 2:

Event 3:

Event 4: 
NON-PHYSICAL VIOLENCE

Questions 47 through 72 pertain to non-physical violence directed at you: work-related threats, sexual harassment, verbal abuse, and bullying.

A threat occurs when someone uses words, gestures, or actions with the intent of intimidating, frightening, or causing harm to you (physically or otherwise). Threats may also include theft or property damage.

Sexual harassment occurs when you experience any type of unwelcome sexual behavior (words or actions) that creates a hostile work environment.

Verbal abuse occurs when someone yells or swears at you, calls you names, or uses other words intended to control or hurt you.

Bullying is defined as repeated acts of intimidation or coercion.

47. Did you experience any work-related threats, sexual harassment, verbal abuse, or bullying, according to the above definitions, within the 12 months prior to today's date? Check one.

$1 \square$ Yes $2 \square$ No $\longrightarrow \underline{\text { IF NO, go to Page } 16 .}$

$\downarrow$

Please provide the following information for any threats, sexual harassment, verbal abuse, or bullying that you experienced during the 12 months prior to today's date. This may include either a single event, with one or more persons, or multiple events with one or more persons.

48. Place a check under each type of behavior(s) you have experienced at work.

49. How frequently did each type of behavior(s) occur?

Check one response per column.

11 to 3 times

24 to 9 times

310 to 19 times

420 times or more

50. Over what time period did the behavior(s) occur? Check one response per column.

1 Single event

2 Less than 1 week

31 week to less than 1 month

41 month to less than 3 months

53 months to less than 6 months

66 months to less than 9 months

79 months to 1 year or more

51. Is(are) the behavior(s) still continuing? Check one response per column.

1 Yes

2 No

52. How did the behavior(s) occur (in most situations)?

Check all that apply per column.

1 Directly (Behavior occurred to you or in your presence)

2 Indirectly (Made aware of the behavior by someone else)

\begin{tabular}{|c|c|c|c|}
\hline Threat & $\begin{array}{c}\text { Sexual } \\
\text { Harassment }\end{array}$ & $\begin{array}{c}\text { Verbal } \\
\text { Abuse }\end{array}$ & Bullying \\
\hline $1 \square$ & $1 \square$ & $1 \square$ & $1 \square$ \\
$1 \square$ & $1 \square$ & $1 \square$ & $1 \square$ \\
$2 \square$ & $2 \square$ & $2 \square$ & $2 \square$ \\
$3 \square$ & $3 \square$ & $3 \square$ & $3 \square$ \\
$4 \square$ & $4 \square$ & $4 \square$ & $4 \square$ \\
$1 \square$ & $1 \square$ & $1 \square$ & $1 \square$ \\
$2 \square$ & $2 \square$ & $2 \square$ & $2 \square$ \\
$3 \square$ & $3 \square$ & $3 \square$ & $3 \square$ \\
$4 \square$ & $4 \square$ & $4 \square$ & $4 \square$ \\
$5 \square$ & $5 \square$ & $5 \square$ & $5 \square$ \\
$6 \square$ & $6 \square$ & $6 \square$ & $6 \square$ \\
$7 \square$ & $7 \square$ & $7 \square$ & $7 \square$ \\
& & & \\
$1 \square$ & $1 \square$ & $1 \square$ & $1 \square$ \\
$2 \square$ & $2 \square$ & $2 \square$ & $2 \square$ \\
$1 \square$ & $1 \square$ & $1 \square$ & $1 \square$ \\
$2 \square$ & $2 \square$ & $2 \square$ & $2 \square$
\end{tabular}


53. What was the nature of the behavior(s) (in most

situations)? Check all that apply per column.

I Physical (gesture, inappropriate touch, facial expression, etc.)

2 Verbal (telephone, etc.)

${ }_{3}$ Graphic (picture, writing, email, webpage, etc.)

4 Theft/Destruction of property

s Other

Specify

54. What was your relationship with the person(s) who threatened/sexually harassed/verbally abused/bullied you (in most situations)? Check all that apply per column.

1 Your current student

2 Your former student

3 Another currently enrolled student

4 Another formerly enrolled student

5 Someone you supervise

6 Another employee

7 Parent

8 Visitor

9 Trespasser

10 No professional relationship

11 Other

88 Unsure

Specify

55. What was(were) the gender(s) of the person(s) who threatened/sexually harassed/verbally abused/bullied you (in most situations)? Check all that apply per column. i Male

2 Female

8 Unsure

56. Was the race/ethnicity of the person(s) (in most situations) the same as your own? Check all that apply per column.

1 Yes

2 No

3 Unsure

\begin{tabular}{|c|c|c|c|}
\hline Threat & $\begin{array}{c}\text { Sexual } \\
\text { Harassment }\end{array}$ & $\begin{array}{l}\text { Verbal } \\
\text { Abuse }\end{array}$ & Bullying \\
\hline 10 & $1 \square$ & $1 \square$ & $1 \square$ \\
\hline $2 \square$ & $2 \square$ & $2 \square$ & $2 \square$ \\
\hline $3 \square$ & $3 \square$ & $3 \square$ & $3 \square$ \\
\hline $4 \square$ & $4 \square$ & $4 \square$ & $4 \square$ \\
\hline $5 \square$ & $5 \square$ & $5 \square$ & $5 D$ \\
\hline 10 & $1 \square$ &,$\square$ & $1 \square$ \\
\hline $2 \square$ & $2 \square$ & $2 \square$ & $2 \square$ \\
\hline $3 \square$ & 30 & $3 \square$ & $3 \square$ \\
\hline $4 \square$ & $4 \square$ & $4 \square$ & $4 \square$ \\
\hline $5 \square$ & $5 \square$ & $5 \square$ & $5 \square$ \\
\hline $6 \square$ & $6 \square$ & $6 \square$ & $6 \square$ \\
\hline $7 \square$ & $7 \square$ & $7 D$ & $7 \square$ \\
\hline $8 \square$ & $8 \square$ & $8 \square$ & $8 \square$ \\
\hline $9 \square$ & $9 \square$ & $9 \square$ & $9 \square$ \\
\hline $10 \square$ & $10 \square$ & $10 \square$ & $10 \square$ \\
\hline $11 \square$ & $11 \square$ & 110 & $11 \square$ \\
\hline $88 \square$ & $88 \square$ & $88 \square$ & $88 \square$ \\
\hline $1 \square$ & $1 \square$ & $1 \square$ & 10 \\
\hline $2 \square$ & $2 \square$ & $2 \square$ & $2 \square$ \\
\hline $8 \square$ & $8 \square$ & $8 \square$ & 80 \\
\hline $1 \square$ & $1 \square$ & 10 & $1 \square$ \\
\hline $2 \square$ & $2 \square$ & $2 \Pi$ & $2 \Pi$ \\
\hline $3 \square$ & $3 \square$ & $3 \square$ & $3 \square$ \\
\hline $1 \square$ & $1 \square$ & 15 & 1 . \\
\hline $2 \square$ & $2 \square$ & $2 \square$ & $2 \square$ \\
\hline 3[] & $3 \square$ & $3 \square$ & $3 \square$ \\
\hline $4 \square$ & $4 \square$ & $4 \square$ & $4 \square$ \\
\hline $8 \square$ & $8 \square$ & $8 \square$ & $8 \square$ \\
\hline $1 \square$ & $1 \square$ & 10 & $1 \square$ \\
\hline $2 \square$ & $2 \square$ & $2 \square$ & $2 \square$ \\
\hline $3 \square$ & $3 \square$ & $3 \square$ & $3 \square$ \\
\hline $\begin{array}{l}4 \square \\
5 \square\end{array}$ & $\begin{array}{l}4 \square \\
5 \square\end{array}$ & $\begin{array}{l}4 \square \\
5 \square\end{array}$ & $4 \square$ \\
\hline & & 5 & $5 \square$ \\
\hline
\end{tabular}

57. In what age group(s) was(were) this(these) person(s) (in most situations)? Check all that apply per column.

I Age less than 13 years

213 years to less than 16 years

316 years to less than 18 years

4 Adult

8 Unsure

58. Was(were) this(these) person(s) impaired (in most

situations)? Check all that apply per column.

1 Yes, because of injury or illness (physical impairment, hearing impairment, etc.)

2 Yes, because of disability (learning, emotional, autism, etc.) or developmental delay

3 Yes, under influence of alcohol, aerosols, or drugs (prescribed or non-prescribed)

4 Not impaired

5 Unsure 
59. Was(were) the behavior(s) witnessed? Check one response per column.

1 Yes, at least once

2 No, never witnessed

8 Unsure

60. As a result of the behavior(s) what types of symptoms and feelings have you experienced? Check all that apply per column.

1 Anger

2 Depression

3 Sadness

${ }_{4}$ Fatigue

5 Frustration

6 Fear/anxiety/stress

7 Shame/Low self-esteem/Low self-confidence

8 Stress-related physical symptoms (headaches, stomach problems, etc.)

9 Difficulty sleeping

10 Nightmares

11 Hallucinations

12 Flashbacks

13 Difficulty concentrating

14 Irritability

15 Intrusive thoughts about the event

16 Hyperarousal/Hypervigilance/Overly cautious

17 Avoidance of any reminders of the event

18 Resurfacing memories of previous trauma

19 Withdrawal from contact with people

20 Other,

21 None

$$
\text { Specify }
$$

61. Did any of your symptoms (identified in Question 60) last

for at least 1 month? Check one response per column.

1 Yes

2 No

31 month has not passed since the event occurred

62. Did any of your symptoms (identified in Question 60) last for 6 months or more? Check one response per column. 1 Yes

2 No

36 months have not passed since the event occurred

63. Are you currently experiencing any persistent problems or symptoms related to the behavior(s)? Check one response per column.

1 Yes

2 No

If YES, please list problems or symptoms:

\begin{tabular}{|c|c|c|c|}
\hline Threat & $\begin{array}{c}\text { Sexual } \\
\text { Harassment }\end{array}$ & $\begin{array}{l}\text { Verbal } \\
\text { Abuse }\end{array}$ & Bullying \\
\hline $\begin{array}{l}1 \square \\
2 \square \\
8 \square\end{array}$ & $\begin{array}{l}1 \square \\
2 \square \\
8 \square\end{array}$ & $\begin{array}{ll}1 & \square \\
2 & \square \\
8 & \square\end{array}$ & $\begin{array}{l}1 \quad \square \\
2 \square \\
8 \square\end{array}$ \\
\hline 10 & 10 & 10 & $1 \square$ \\
\hline $2 \square$ & $2 \square$ & $2 \square$ & $2 \square$ \\
\hline $3 \square$ & $3 \square$ & 3[ & $3 \square$ \\
\hline $4 \square$ & $4 \square$ & $4 \square$ & $4 \square$ \\
\hline $5 \square$ & $s \square$ & $5 \square$ & 50 \\
\hline $6 \square$ & $6 \square$ & $6 \square$ & 6] \\
\hline $7 \square$ & $7 \square$ & $7 \square$ & $7 \square$ \\
\hline $8 \square$ & $8 \square$ & $8 \square$ & $8 \square$ \\
\hline $9 \square$ & $9 \square$ & $9 \square$ & $9 \square$ \\
\hline 10] & $10 \square$ & $10 \square$ & $10 \square$ \\
\hline 11$]$ & $11 \square$ & $11 \square$ & 11匹 \\
\hline 127 & $12 \square$ & $12 \square$ & $12 \square$ \\
\hline $13 \square$ & 13[] & $13 \square$ & $13 \square$ \\
\hline $14 \square$ & $14 \square$ & $14 \square$ & $14 \square$ \\
\hline $15 \square$ & 15[ & $15 \square$ & 15[ \\
\hline 167 & $16 \div$ & 167 & $16 \sqcap$ \\
\hline $17 \square$ & $13 \square$ & $17 \square$ & 17! \\
\hline $18 \square$ & $18 \square$ & $18 \square$ & $18 \square$ \\
\hline $19 \square$ & $19 \square$ & $19 \square$ & $19 \square$ \\
\hline $20 \mathrm{~L}$ & $20 \square$ & $20 \square$ & $20 \square$ \\
\hline $21 \square$ & 210 & 210 & $21 \square$ \\
\hline 10 & 1ㅁ & 10 & 10 \\
\hline $2 \square$ & $2 \square$ & $2 \square$ & $2 \square$ \\
\hline $3 \square$ & $3 \square$ & $3 \square$ & $3 \square$ \\
\hline $1 \square$ & $1 \square$ & $1 \square$ & 10 \\
\hline $2 \square$ & $2 \square$ & $2 \square$ & $2 \square$ \\
\hline $3 \square$ & $3 \square$ & $3 \square$ & $3 \square$ \\
\hline 10 & $1 \square$ & $1 \square$ & $1 \square$ \\
\hline $2 \square$ & $2 \square$ & $2 \square$ & $2 \square$ \\
\hline
\end{tabular}


64. How would you rate the severity of your symptom(s)/ problem(s) resulting from the behavior(s)? Consider any limitation of your abilities (physical/mental) and/or activities. Check one response per column.

1 No limitations of abilities/activities

2 Some limitations of abilities/activities

3 Moderate limitations of abilities/activities

4 Severe limitations of abilities/activities

5 Disabling-inability to function

65. Were you treated by any of the following as a result of the behavior(s)? Check all that apply per column.

i No treatment

2 Physician (non-Psychiatrist)

${ }_{3}$ Chiropractor

4 Nurse/Nurse Practitioner/Nurse Clinician/ Physician's Assistant

5 Psychiatrist/ Psychologist/Therapist

6 Homeopathic, Alternative, or Non-traditional medicine provider

7 Treated yourself

8 Other

Specify

66. Were you admitted to a hospital as a result of the behavior(s)? Check one response per column.

IYes

2 No

If YES, for how many days? (if none, fill in 0 )

67. What changes in your work situation have occurred as a result of the behavior(s)? Check all that apply per column. 1 Quit your job

2 Voluntary transfer to another location

3 Involuntary transfer to another location

4 Leave of absence

5 Restriction/modification of work activities

6 No changes

7 Other

$$
\text { Specify }
$$

68. How long were regular work and/or non-work activities restricted as a result of the behavior(s)? Check one response per column.

I Less than 4 hours

24 hours to less than 1 day

31 day to less than 3 days

43 days to less than 7 days

57 days to less than 14 days

614 days to less than 1 month

71 month to less than 3 months

83 months or more

9 No restriction

\begin{tabular}{|c|c|c|c|}
\hline Threat & $\begin{array}{c}\text { Sexual } \\
\text { Harassment }\end{array}$ & $\begin{array}{l}\text { Verbal } \\
\text { Abuse }\end{array}$ & Bullying \\
\hline $1 \square$ & $1 \square$ & $1 \square$ & $1 \square$ \\
\hline $2 \square$ & $2 \square$ & $2 \square$ & $2 \square$ \\
\hline $3 \square$ & $3 \square$ & $3 \square$ & $3 \square$ \\
\hline $4 \square$ & $4 \square$ & $4 \square$ & $4 \square$ \\
\hline $5 \square$ & $5 \square$ & $5 \square$ & $5 \square$ \\
\hline 10 & 10 & $1 \square$ & $1 \square$ \\
\hline $2 \square$ & $2 \square$ & $2 \square$ & $2 \square$ \\
\hline $3 \square$ & $3 \square$ & $3 \square$ & $3 \square$ \\
\hline $4 \square$ & $4 \square$ & $4 \square$ & $4 \square$ \\
\hline $5 \square$ & $5 \square$ & $5 \square$ & $5 \square$ \\
\hline $6 \square$ & $6 \square$ & $6 \square$ & $6 \square$ \\
\hline $7 \square$ & $7 \square$ & $7 \square$ & $7 \square$ \\
\hline $8 \square$ & $8 \square$ & $8 \square$ & $8 \square$ \\
\hline $1 \square$ & $1 \square$ & $1]$ & 10 \\
\hline $2 \square$ & $2 \square$ & $2 \square$ & $2 \square$ \\
\hline days & days & days & days \\
\hline $1 \square$ & 1[ & 10 & 10 \\
\hline $2 \square$ & $2 \square$ & $2 \square$ & $2 \square$ \\
\hline $3 \square$ & $3 \square$ & $3 \square$ & $3 \square$ \\
\hline $4 \square$ & $4 \square$ & $4 \square$ & $4 \square$ \\
\hline $5 \mathbb{D}$ & $5 \square$ & $5 \square$ & $5 \square$ \\
\hline $6 \square$ & $6 \square$ & $6 \square$ & $6 \square$ \\
\hline $7 \square$ & $7 \square$ & $7 \square$ & $7 \square$ \\
\hline 10 & $1 \square$ & $1 \square$ & $1 \square$ \\
\hline $2 \square$ & $2 \square$ & $2 \square$ & $2 \square$ \\
\hline $3 \square$ & $3 \square$ & $3 \square$ & 3ए \\
\hline $4 \square$ & $4 \square$ & $4 \square$ & $4 \square$ \\
\hline $5 \square$ & $5 \square$ & $5 \square$ & $5 \square$ \\
\hline $6 \square$ & $6 \square$ & $6 \square$ & $6 \square$ \\
\hline $7 \square$ & $7 \square$ & $7 \square$ & $7 \square$ \\
\hline $8 \square$ & $8 \square$ & $8 \square$ & $8 \square$ \\
\hline $9 \square$ & $9 \square$ & $9 \square$ & $9 \square$ \\
\hline
\end{tabular}


69. Are your regular activities still restricted as a result of the behavior(s)? Check one response per column.

1 Yes

2 No

70. As a result of the behavior(s), how many days were you absent from work? Check one response per column.

1 Less than 4 hours

24 hours to less than 1 day

31 day to less than 3 days

43 days to less than 7 days

57 days to less than 14 days

614 days to less than 1 month

71 month to less than 3 months

83 months or more

9 No absence

71. In general, did you report the behavior(s) to

administration? Check all that apply per column.

1 Yes, orally

2 Yes, written

3 No

If YES, in your opinion, was administration adequately responsive to your report?

4 Yes

5 No

6 Unsure

If $\underline{\mathrm{NO}}$, please check reason(s) that event(s) was(were) not reported.

8 Reporting takes too much time

9 Unaware of reporting process

10 Did not expect report to change situation

11 Did not feel necessary to report

12 Other

$$
\text { Specify }
$$

\begin{tabular}{|c|c|c|c|}
\hline Threat & $\begin{array}{c}\text { Sexual } \\
\text { Harassment }\end{array}$ & $\begin{array}{l}\text { Verbal } \\
\text { Abuse }\end{array}$ & Bullying \\
\hline $1 \square$ & $1 \square$ & $1 \square$ & $1 \square$ \\
\hline $2 \square$ & $2 \square$ & $2 \square$ & $2 \square$ \\
\hline $1 \square$ & 10 & 10 & $1 \square$ \\
\hline $2 \square$ & $2 \square$ & $2 \square$ & $2 \square$ \\
\hline $3 \square$ & $3 \square$ & $3 \square$ & $3 \square$ \\
\hline $4 \square$ & $4 \square$ & $4 \square$ & $4 \square$ \\
\hline $5 \square$ & $5 \square$ & $5 \square$ & $5 \square$ \\
\hline $6 \square$ & $6 \square$ & $6 \square$ & $6 \square$ \\
\hline $7 \square$ & $7 \square$ & $7 \square$ & $7 \square$ \\
\hline $8 \square$ & $8 \square$ & $8 \square$ & $8 \square$ \\
\hline $9 \square$ & $9 \square$ & $9 \square$ & $9 \square$ \\
\hline $1 \square$ & $1 \square$ & 10 & $1 \square$ \\
\hline $2 \square$ & $2 \square$ & $2 \square$ & $2 \square$ \\
\hline 3[ & $3 \square$ & $3 \square$ & 3[ \\
\hline $4 \square$ & $4 \square$ & $4 \square$ & $4 \square$ \\
\hline $5 \square$ & $5 \square$ & $5 \square$ & $5 \square$ \\
\hline $6 \square$ & $6 \square$ & $6 \square$ & $6 \square$ \\
\hline $8 \square$ & $8 \square$ & $8 \square$ & $8 \square$ \\
\hline $9 \square$ & $9 \square$ & $9 \square$ & $9 \square$ \\
\hline $10 \square$ & $10 \square$ & $10 \square$ & $10 \square$ \\
\hline $11 \square$ & $11 \square$ & $11 \square$ & $11 \square$ \\
\hline $12 \square$ & $12 \square$ & $12 \square$ & $12 \square$ \\
\hline
\end{tabular}

Comments regarding reporting:

72. Please describe how the behavior(s) occurred, in general. What were you typically doing just prior to the event, and what was the person who threatened/sexually harassed/verbally abused/bullied you doing? What triggered the behavior(s)? Please use extra paper if necessary.

Threat:

Sexual Harassment:

Verbal Abuse:

Bullving: 
*** Please answer the following questions regardless of your personal experience with violence. * 73. In the 12 months prior to today's date, how often have you witnessed other licensed educators being:

a. physically assaulted in your work environment? Check one.

1 $\square$ Never $\quad 2 \square 1-3$ times $\quad 3 \square 4$-10 times $\quad 4 \square$ More than 10 times

b. threatened in your work environment? Check one.

$1 \square$ Never $\quad 2 \square 1-3$ times $\quad 3 \square 4-10$ times $\quad 4 \square$ More than 10 times

c. sexually harassed in your work environment? Check one.

$1 \square$ Never $\quad 2 \square 1-4$ times $\quad 3 \square 5$-9 times $\quad 4 \square 10-19$ times $5 \quad \square 20$ times or more

d. verbally abused in your work environment? Check one.

$1 \square$ Never $\quad 2 \square 1-4$ times $\quad 3 \square 5$-9 times $\quad 4 \square 10$ - 19 times $5 \quad \square 20$ times or more

e. bullied in your work environment? Check one.

$1 \square$ Never $\quad 2 \square 1-4$ times $\quad 3 \square 5-9$ times $\quad 4 \square 10$ - 19 times $5 \quad \square 20$ times or more

74. In the 12 months prior to today's date, how often were you made aware of, but not a witness to, other licensed educators being:

a. phvsically assaulted in your work environment? Check one.

$1 \square$ Never $\quad 2 \square 1-3$ times $\quad 3 \square$ 4-10 times $\quad 4 \square$ More than 10 times

b. threatened in your work environment? Check one.

$1 \square$ Never $\quad 2 \square 1-3$ times $\quad 3 \square 4$-10 times $\quad 4 \square$ More than 10 times

c. sexually harassed in your work environment? Check one.

1 $\square$ Never $\quad 2 \square$ 1-4 times $\quad 3 \square$ 5-9 times $\quad 4 \square 10-19$ times $5 \quad \square 20$ times or more

d. verbally abused in your work environment? Check one.

$1 \square$ Never $\quad 2 \square 1-4$ times $\quad 3 \square$ 5-9 times $\quad 4 \square 10-19$ times $5 \quad \square 20$ times or more

e. bullied in your work environment? Check one.

$1 \square$ Never $\quad 2 \square$ - 4 times $\quad 3 \square 5$-9 times $\quad 4 \square 10$ - 19 times $5 \quad \square 20$ times or more

75. Do you believe that work-related violence against licensed educators is a problem in your work environment? Check one.

$1 \square$ Yes $\quad 2 \square$ No $\quad 8 \square$ Unsure

76. Do you believe that work-related violence against licensed educators can be prevented? Check one.

$1 \square$ Yes $\quad 2 \square$ No $\quad 8 \square$ Unsure

Comments: 
77. As of today's date, how many years have you worked as a licensed educator in any location? Please indicate the number of years and/or months.

year(s) month(s)

78. As of today's date, what is your highest level of education? Check one.
I $\square$ Associate Degree
$3 \square$ Master's Degree
$\$ \square$ Doctorate Degree
$2 \square$ Bachelor's Degree
$4 \square$ Education Specialist Degree

79. What is your gender? Check one.

$1 \square$ Male $\quad 2 \square$ Female

80. What is your date of birth? Please write in date.

(month/day/year)

81. Which of the following best describes your ethnic background? Check one.

$1 \square$ Hispanic $2 \square$ Not Hispanic

82. Which of the following best describes your race? Check all that apply.

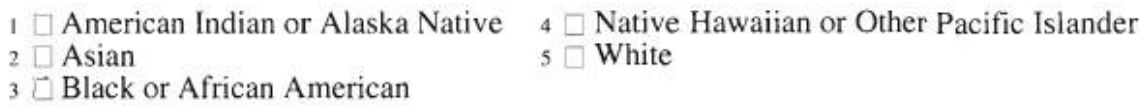

83. What is your current marital status? Check one.
$1 \square$ Married
$2 \square$ Living as married
$5 \square$ Separated
6 Divorced

$\square$ Never married
We would appreciate your providing a telephone number in case we need to clarify some information with you.
( $1 \square$ work $2 \square$ home $3 \square$ cell
area code

***Thank you for taking the time to participate in this important study!*** Please return your questionnaire in the enclosed envelope.

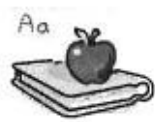

